\title{
PROBING EXTRAGALACTIC DUST THROUGH NEARBY GAMMA-RAY BURST AFTERGLOWS
}

\author{
S. L. LIANG ${ }^{1,2}$ AND AIGEN LI $^{2}$ \\ ${ }^{1}$ Department of Physics, Hunan Normal University, Changsha 410071, China; shunlinliang@ @izzou.edu \\ 2 Department of Physics and Astronomy, University of Missouri, Columbia, MO 65211, USA; lia @ missouri.edu \\ Received 2009 September 14; accepted 2009 December 30; published 2010 January 20
}

\begin{abstract}
The quantities and wavelength dependencies of the dust extinction along the lines of sight toward 33 nearby gammaray bursts (GRBs) with redshifts $z<2$ are derived from fitting their afterglow spectral energy distributions. Unlike previous studies which often assume a specific extinction law like that of the Milky Way (MW) and the Large and Small Magellanic Clouds (LMC/SMC), our approach—we call it the "Drude" approach—is more flexible in determining the true wavelength dependence of the extinction (while the shape of the extinction curve inferred from that relying on a priori assumption of a template extinction law is, of course, fixed). The extinction curves deduced from the Drude approach display a wide diversity of shapes, ranging from relatively flat curves to curves which are featureless and steeply rise toward the far-ultraviolet, and from curves just like that of the MW, LMC, and SMC to curves resembling that of the MW and LMC but lacking the $2175 \AA$ bump. The visual extinction $A_{V}$ derived from the Drude approach is generally larger by a factor of $\sim 2-5$ than that inferred by assuming a SMC-type template extinction law. Consistent with previous studies, the extinction-to-gas ratio is mostly smaller than that of the MW, and does not seem to correlate with the shape of the extinction curve. It is shown that the standard silicate-graphite interstellar grain model closely reproduces the extinction curves of all 33 GRBs host galaxies. For these 33 bursts at $z<2$, we find no evidence for the evolution of the dust extinction, dust sizes, and relative abundances of silicate to graphite on redshifts.
\end{abstract}

Key words: dust, extinction - gamma rays: general

Online-only material: color figures

\section{INTRODUCTION}

The presence of dust in the host galaxies of gamma-ray burst (GRB) is now well recognized (see Li et al. 2008a for a summary), as revealed by dark bursts, ${ }^{3}$ reddening of GRB afterglows (Klose et al. 2000; Levan et al. 2006), depletion of dust-forming heavy elements (Savaglio et al. 2003), thermal emission of GRB host galaxies in the infrared (IR) and submillimeter wavelengths (Michałowski et al. 2008, 2009), and the association of longduration bursts with the death of young massive stars (which suggests the residence of long bursts in dense, dusty star-forming regions; see Woosley \& Bloom 2006 for a review). Very recently, Berger et al. (2009) reported the discovery of a very red nearIR and optical afterglow of GRB 070724A, a short-duration GRB. They argued that dust extinction (with an visual extinction of $A_{V} \approx 2 \mathrm{mag}$ ) may also be responsible for reddening the optical/near-IR afterglow of this short burst (which is much redder than expected in the standard afterglow model).

However, little is known regarding the quantities and properties of the dust in GRB host galaxies. While studies based on fitting the spectral energy distributions (SEDs) of afterglows in the ultraviolet (UV), visible and near-IR with a power law (or broken power laws) reddened by a known template extinction law such as that of the Milky Way (MW) and the Large/Small Magellanic clouds (LMC/SMC) often suggest little extinction and a SMC-type extinction law (e.g., see Kann et al. 2006), dust

\footnotetext{
3 Dark bursts are those events with no detected optical afterglow or with detected optical flux significantly suppressed compared with that expected from the observed X-ray afterglow (Groot et al. 1998; Jakobsson et al. 2004; van der Horst et al. 2009). Even in the Swift era, despite deep and prompt searches, an appreciable fraction $(\sim 50 \%)$ of the bursts are identified as dark (e.g., see Melandri et al. 2008; Cenko et al. 2009; Perley et al. 2009; Zheng et al. 2009). The optical darkness of GRB afterglows is largely attributed to the extinction of dust local to the GRB host galaxies (Cenko et al. 2009; Perley et al. 2009).
}

depletion studies often favor large extinction and a flat or even gray extinction law (e.g., see Savaglio et al. 2003; Savaglio \& Fall 2004), in stark contrast to the featureless SMC extinction law which steeply rises with inverse wavelength $\left(\lambda^{-1}\right)$. But some studies based on SED modeling also suggest that large extinction is not uncommon. For some bursts, the visual extinction $A_{V}$ exceeds several magnitudes, e.g., $A_{V} \approx 3.2 \mathrm{mag}$ for GRB 080607 (Prochaska et al. 2009), $A_{V}>3.8 \mathrm{mag}$ for GRB 970828 (Djorgovski et al. 2001), $2 \lesssim A_{V} \lesssim 5$ mag for GRB 060923A (Tanvir et al. 2008), $A_{V}>5.0 \mathrm{mag}$ for GRB 061222A (Perley et al. 2009), $A_{V} \approx 5.5 \mathrm{mag}$ for GRB 070306 (Jaunsen et al. 2008), and $A_{V}>5.5 \mathrm{mag}$ for GRB 070521 (Perley et al. 2009), just to list a few. Furthermore, extinction laws very different from that of the SMC have been inferred for some bursts. The $2175 \AA$ extinction bump, the most prominent UV extinction feature in the Galactic extinction law, is clearly seen in GRB 070802 (Krühler et al. 2008; Elíasdóttir et al. 2009; Liang \& Li 2009), GRB 080607 (Prochaska et al. 2009), and probably in GRB 970508 (Stratta et al. 2004), GRB 991216 (Kann et al. 2006; Vreeswijk et al. 2006), GRB 050802 (Schady et al. 2007), and GRB 050904 (Liang \& Li 2009). On the other hand, some bursts appear to have a featureless extinction law but much flatter than that of the SMC (e.g., see Chen et al. 2006; Li et al. 2008b; Perley et al. 2008a).

In view of the wide diverse nature of the quantity and wavelength dependence of the dust extinction of GRB host galaxies, we developed a technique (we call it the "Drude" approach) to fit the observed afterglow SEDs to derive the extinction and its wavelength dependence of the dust local to GRB hosts (see Li et al. 2008a). The advantage of this approach is that it does not require a priori assumption of a template extinction law (see Section 2). This is important as there is no reason to believe that the extinction curves of GRB host galaxies which exhibit a wide range of ages, luminosities, metallicities, 
and star formation rates should resemble that of the known MW, LMC, SMC, and other templates. Even in the MW, there does not exist a universal extinction law: the extinction curve varies from one sightline to another. In the SMC and the LMC, there are also regional variations (see Gordon et al. 2003). So far, only a few other galaxies have their extinction curves measured; the extinction curves of these external galaxies differ much from the standard MW, LMC, and SMC laws (see Section 2.2 of Draine 2003). The "Calzetti" starburst attenuation law (i.e., the internal extinction by dust in starburst galaxies) determined by Calzetti et al. (1994) and the extinction curve inferred for a distant broad absorption line (BAL) QSO at a redshift $z \approx 6.2$ by Maiolino et al. (2004) $)^{4}$ do not look like that of the MW, LMC, or SMC at all. Apparently, we should not expect a universal extinction law for GRB host galaxies.

Adopting the "Drude" approach (Li et al. 2008a; Section 2), in this work we perform a systematic study of the dust extinction of the host galaxies for a sample of 33 nearby or low-redshift (with $z<2$ ) GRBs. In a separate paper, we will explore highredshifted GRB host galaxies (at $z>2$ ) with an aim of studying the properties of interstellar dust of the early universe and investigating whether (and how) they vary with $z$ (S. L. Liang \& A. Li 2010, in preparation). We compile the SEDs in the UV/ optical/near-IR bands of these GRB afterglows (Section 3), and determine both the quantity and wavelength-dependence of the extinction for the host galaxy of each GRB (Section 3). In Section 4, we discuss the deduced extinction curves and model them in terms of the silicate-graphite interstellar dust model. The dust properties and how the dust is correlated with gas are also discussed in Section 4. The major conclusion of this work is summarized in Section 5.

\section{DETERMINING THE DUST EXTINCTION: THE DRUDE APPROACH}

We characterize the dust extinction properties of GRB hosts with the rest-frame visual extinction $A_{V}$ and the wavelengthdependence of the extinction (i.e., $A_{\lambda} / A_{V}$ or $A_{v} / A_{V}$ if expressed in frequency $v$, often known as the "extinction curve" or "extinction law"). We derive $A_{V}$ and $A_{\lambda} / A_{V}$ (or $A_{\nu} / A_{V}$ ) by fitting the UV, optical, and near-IR afterglow photometry with a dust-reddened power-law model through

$$
F_{\nu}=F_{\mathrm{o}}(v / \mathrm{Hz})^{-\beta} \exp \left[-\frac{A_{V}}{1.086} \frac{A_{(1+z) v}}{A_{V}}\right],
$$

where $F_{v}$ is the afterglow photometry (with the Galactic foreground extinction corrected), $\beta$ is the intrinsic power-law slope of the afterglow, $F_{\mathrm{o}}$ is a normalization constant, $A_{(1+z) v}$ is the rest-frame extinction, and $z$ is the GRB redshift.

As described in Li et al. (2008a), the "Drude" approach approximates the wavelength-dependence of the extinction by a simple formula consisting of four dimensionless parameters $\left(c_{1}, c_{2}, c_{3}\right.$, and $\left.c_{4}\right)$

$$
\begin{aligned}
A_{\lambda} / A_{V}= & \frac{c_{1}}{(\lambda / 0.08)^{c_{2}}+(0.08 / \lambda)^{c_{2}}+c_{3}} \\
& +\frac{233\left[1-c_{1} /\left(6.88^{c_{2}}+0.145^{c_{2}}+c_{3}\right)-c_{4} / 4.60\right]}{(\lambda / 0.046)^{2}+(0.046 / \lambda)^{2}+90} \\
& +\frac{c_{4}}{(\lambda / 0.2175)^{2}+(0.2175 / \lambda)^{2}-1.95}
\end{aligned}
$$

\footnotetext{
4 Stratta et al. (2007) successfully reproduced the observed afterglow SED of GRB 050904 at $z \approx 6.29$ with the extinction curve of this high-redshift BAL QSO (but also see Liang \& Li 2009).
}

where $\lambda$ is in $\mu \mathrm{m}$. In Equation (2), the first term in the righthand side represents the far-UV extinction rise, the second term and the third term, respectively, account for the near-IR/visible extinction and the $2175 \AA$ extinction bump.

We use the Levenberg-Marquardt minimization algorithm (Press et al. 1992) to find the best-fit parameters $c_{j}(j=1, \ldots, 4)$ and $\beta, A_{V}$ and $F_{\mathrm{o}}$. To be complete, we also model the afterglow SEDs using the MW, LMC, and SMC template extinction laws. Since for a given template the wavelength dependence of the extinction $A_{\lambda} / A_{V}$ is fixed, we only need to determine three parameters: $\beta, A_{V}$, and $F_{\mathrm{o}}$.

Compared to models based on template extinction curves, the "Drude" model is preferred because (1) it eliminates the need for a priori assumption of template laws (i.e., we do not need to choose a presumed dust extinction model), and (2) the analytical formula (Equation (2)) on which the "Drude" model is based restores the widely adopted MW, SMC, LMC, and "Calzetti" templates-even if the "true" extinction curve of a GRB host happens to resemble a certain template law, the "Drude" approach will allow us to restore it (see Li et al. 2008a).

We should note that it is unfortunate that the Drude approach needs more free parameters ${ }^{5}$-but this is the nature of the Drude approach: it allows the extinction curve to have a flexible shape, one has to have parameters to control the shape variation. While the approach of using "template" extinction curves has fewer parameters, their extinction shapes are fixed (although it is well recognized that there is no prior reason why a GRB host should have an extinction shape like that of a fixed template). Note that the Cardelli et al. (1989, hereafter CCM) parameterization of interstellar extinction curves with a single parameter $R_{V}{ }^{6}$ (Cardelli et al. 1989) is only valid for the Galactic sightlinesindeed, it does not even apply to the LMC and the SMC (Gordon et al. 2003), ${ }^{7}$ otherwise one could simply adopt the CCM formula which has only one parameter (i.e., $R_{V}$ ) instead of Equation (2) which has four parameters. The Fitzpatrick \& Massa (1990, "FM") formula consisting of four parameters is good for the MW, SMC, and LMC; but it is only valid for $\lambda<0.3 \mu \mathrm{m}$.

\section{RESULTS}

To investigate the wavelength-dependence of dust extinction in the UV/optical/near-IR of the comoving frame and its effects on the afterglow SEDs, one needs to select the samples which have the necessary wavelength coverage for determining the dust extinction. The foreground (Galactic) extinction has been corrected for each burst. For those observations taken

\footnotetext{
5 Due to the sparsity of their photometric data, for some bursts the number of model parameters $N_{\text {para }}$ exceeds the number of data points $N_{\text {obs }}$. For this reason, we measure the goodness of a model fit in terms of $\chi^{2} / N_{\text {obs }}$ (see Tables 1 and 2). Future studies with the seven-band GROND imaging instrument and other photometric (Greiner et al. 2008) and even spectroscopic instruments (e.g., the FORS2 spectrograph on the Very Large Telescope (VLT); see Fynbo et al. 2007) will allow us to place better constraints on model parameters.

${ }_{6} R_{V} \equiv A_{V} / E(B-V)$ is the total-to-selective extinction ratio, where $E(B-V) \equiv A_{B}-A_{V}$, the color excess, is the difference between the extinction in $B$ - and $V$ bands.

7 The total-to-selective extinction ratio of the SMC bar is $R_{V} \approx 2.87$ (Gordon \& Clayton 1998). The extinction curve of the SMC bar differs substantially from that predicted from the CCM formula with $R_{V}=2.87$ : while the CCM $R_{V}=2.87$ curve has a moderately strong $2175 \AA$ bump and a slightly steeper far-UV extinction rise than the MW average (with $R_{V} \approx 3.1$ ), the SMC extinction is featureless and steeply rises toward the far-UV. The LMC extinction law is also substantially different from that predicted from the CCM formula with $R_{V} \approx 2.6$, a value obtained by averaging over $10 \mathrm{LMC}$ regions (Misselt et al. 1999).
} 
Table 1

Parameters for Fitting the Afterglow SEDs with the MW, LMC, and SMC Template Extinction Laws

\begin{tabular}{|c|c|c|c|c|c|c|c|c|c|c|c|c|}
\hline \multirow[t]{2}{*}{ GRB } & \multicolumn{4}{|c|}{ MW Dust } & \multicolumn{4}{|c|}{ LMC Dust } & \multicolumn{4}{|c|}{ SMC Dust } \\
\hline & $A_{V}(\mathrm{mag})$ & $\beta$ & $\begin{array}{c}\log F_{\mathrm{o}} \\
(\mu \mathrm{Jy})\end{array}$ & $\chi^{2} / N_{\text {obs }}$ & $A_{V}(\mathrm{mag})$ & $\beta$ & $\begin{array}{c}\log F_{\mathrm{o}} \\
(\mu \mathrm{Jy})\end{array}$ & $\chi^{2} / N_{\text {obs }}$ & $A_{V}(\mathrm{mag})$ & $\beta$ & $\begin{array}{c}\log F_{\mathrm{o}} \\
(\mu \mathrm{Jy})\end{array}$ & $\chi^{2} / N_{\text {obs }}$ \\
\hline 970508 & $0.24 \pm 0.08$ & $0.14 \pm 0.06$ & 3.26 & 0.38 & $0.16 \pm 0.05$ & $0.10 \pm 0.04$ & 3.47 & 2.42 & $0.13 \pm 0.04$ & $0.25 \pm 0.06$ & 5.27 & 1.46 \\
\hline 980703 & $0.72 \pm 0.26$ & $1.73 \pm 0.62$ & 27.22 & 0.65 & $0.21 \pm 0.07$ & $2.25 \pm 0.80$ & 34.74 & 0.91 & $0.39 \pm 0.14$ & $2.05 \pm 0.73$ & 31.87 & 0.80 \\
\hline 990123 & $0.21 \pm 0.13$ & $0.44 \pm 0.10$ & 7.66 & 0.88 & $0.14 \pm 0.06$ & $0.34 \pm 0.08$ & 6.10 & 0.48 & $0.17 \pm 0.08$ & $0.40 \pm 0.06$ & 6.99 & 0.45 \\
\hline 990510 & $0.16 \pm 0.04$ & $0.67 \pm 0.22$ & 11.94 & 2.38 & $0.18 \pm 0.07$ & $0.30 \pm 0.14$ & 6.44 & 0.32 & $0.15 \pm 0.05$ & $0.60 \pm 0.26$ & 10.96 & 0.78 \\
\hline 991208 & $0.86 \pm 0.29$ & $0.16 \pm 0.07$ & 5.65 & 0.08 & $0.78 \pm 0.18$ & $0.17 \pm 0.05$ & 5.78 & 0.10 & $0.90 \pm 0.20$ & $0.11 \pm 0.04$ & 4.95 & 0.07 \\
\hline 991216 & $0.16 \pm 0.06$ & $0.39 \pm 0.12$ & 8.09 & 0.20 & $0.14 \pm 0.03$ & $0.35 \pm 0.12$ & 7.57 & 1.13 & $0.14 \pm 0.05$ & $0.39 \pm 0.14$ & 8.08 & 0.35 \\
\hline 000911 & $0.44 \pm 0.17$ & $0.55 \pm 0.28$ & 10.08 & 0.51 & $0.17 \pm 0.03$ & $0.77 \pm 0.22$ & 13.28 & 0.45 & $0.30 \pm 0.08$ & $0.64 \pm 0.10$ & 11.41 & 0.41 \\
\hline 010222 & $0.15 \pm 0.05$ & $1.04 \pm 0.12$ & 17.63 & 1.99 & $0.13 \pm 0.03$ & $0.81 \pm 0.09$ & 14.12 & 0.67 & $0.16 \pm 0.04$ & $0.96 \pm 0.11$ & 16.43 & 0.98 \\
\hline 010921 & $0.83 \pm 0.27$ & $0.91 \pm 0.29$ & 15.72 & 0.03 & $1.23 \pm 0.39$ & $0.11 \pm 0.03$ & 3.92 & 0.03 & $1.33 \pm 0.43$ & $0.19 \pm 0.06$ & 5.16 & 0.03 \\
\hline 011121 & $0.50 \pm 0.11$ & $0.55 \pm 0.19$ & 9.55 & 0.56 & $0.40 \pm 0.13$ & $0.61 \pm 0.15$ & 10.40 & 0.43 & $0.48 \pm 0.14$ & $0.55 \pm 0.14$ & 9.55 & 0.46 \\
\hline 020405 & $0.16 \pm 0.05$ & $0.96 \pm 0.20$ & 15.35 & 1.07 & $0.17 \pm 0.06$ & $0.93 \pm 0.23$ & 14.98 & 1.02 & $0.20 \pm 0.09$ & $0.91 \pm 0.15$ & 14.63 & 1.02 \\
\hline 020813 & $0.11 \pm 0.05$ & $0.89 \pm 0.16$ & 15.23 & 1.09 & $0.16 \pm 0.04$ & $0.80 \pm 0.20$ & 13.97 & 0.53 & $0.21 \pm 0.08$ & $0.71 \pm 0.15$ & 12.61 & 0.60 \\
\hline 030226 & $0.10 \pm 0.03$ & $0.61 \pm 0.14$ & 10.93 & 3.17 & $0.14 \pm 0.03$ & $0.43 \pm 0.11$ & 8.20 & 0.56 & $0.12 \pm 0.04$ & $0.43 \pm 0.10$ & 8.30 & 1.24 \\
\hline 030328 & $0.18 \pm 0.07$ & $0.41 \pm 0.19$ & 7.87 & 2.36 & $0.15 \pm 0.08$ & $0.20 \pm 0.08$ & 4.71 & 0.40 & $0.15 \pm 0.05$ & $0.31 \pm 0.10$ & 6.44 & 0.52 \\
\hline 030329 & $0.53 \pm 0.23$ & $0.32 \pm 0.10$ & 7.22 & 0.36 & $0.38 \pm 0.14$ & $0.43 \pm 0.15$ & 8.78 & 0.34 & $0.49 \pm 0.17$ & $0.34 \pm 0.13$ & 7.50 & 0.36 \\
\hline 040924 & $0.26 \pm 0.06$ & $0.55 \pm 0.16$ & 8.60 & 0.84 & $0.21 \pm 0.04$ & $0.58 \pm 0.16$ & 9.03 & 0.97 & $0.29 \pm 0.06$ & $0.51 \pm 0.15$ & 8.03 & 0.80 \\
\hline 041006 & $0.12 \pm 0.03$ & $0.39 \pm 0.13$ & 7.52 & 0.42 & $0.13 \pm 0.02$ & $0.36 \pm 0.12$ & 7.16 & 0.47 & $0.15 \pm 0.02$ & $0.36 \pm 0.12$ & 7.09 & 0.42 \\
\hline 050318 & $0.93 \pm 0.33$ & $1.74 \pm 0.41$ & 44.06 & 0.60 & $0.65 \pm 0.23$ & $1.80 \pm 0.36$ & 43.93 & 0.24 & $0.79 \pm 0.28$ & $1.73 \pm 0.55$ & 44.01 & 0.29 \\
\hline 050408 & $0.23 \pm 0.04$ & $1.34 \pm 0.22$ & 21.00 & 2.58 & $0.75 \pm 0.12$ & $0.27 \pm 0.04$ & 5.34 & 0.39 & $1.02 \pm 0.17$ & $0.11 \pm 0.02$ & 3.04 & 0.84 \\
\hline 050525A & $0.38 \pm 0.11$ & $1.77 \pm 0.50$ & 28.19 & 0.10 & $0.17 \pm 0.05$ & $1.97 \pm 0.56$ & 31.03 & 0.15 & $1.33 \pm 0.37$ & $0.11 \pm 0.03$ & 3.91 & 0.06 \\
\hline 050824X & $0.30 \pm 0.09$ & $0.16 \pm 0.04$ & 3.79 & 0.35 & $0.34 \pm 0.06$ & $0.14 \pm 0.02$ & 3.08 & 0.17 & $0.35 \pm 0.08$ & $0.14 \pm 0.03$ & 3.08 & 0.21 \\
\hline 051111 & $0.18 \pm 0.05$ & $0.98 \pm 0.25$ & 17.34 & 1.77 & $0.35 \pm 0.09$ & $0.42 \pm 0.11$ & 9.13 & 0.34 & $0.62 \pm 0.16$ & $0.12 \pm 0.03$ & 4.90 & 0.56 \\
\hline 060614 & $0.26 \pm 0.12$ & $0.69 \pm 0.16$ & 12.23 & 0.52 & $0.17 \pm 0.16$ & $0.67 \pm 0.14$ & 11.93 & 0.44 & $0.24 \pm 0.10$ & $0.69 \pm 0.20$ & 12.23 & 0.47 \\
\hline 060729 & $0.20 \pm 0.03$ & $0.83 \pm 0.22$ & 14.45 & 0.52 & $0.16 \pm 0.04$ & $0.87 \pm 0.32$ & 15.05 & 0.44 & $0.16 \pm 0.04$ & $0.87 \pm 0.25$ & 14.98 & 0.56 \\
\hline 061121 & $1.28 \pm 0.52$ & $0.93 \pm 0.38$ & 16.60 & 2.97 & $0.65 \pm 0.26$ & $1.29 \pm 0.52$ & 21.57 & 3.51 & $1.54 \pm 0.62$ & $0.11 \pm 0.04$ & 4.51 & 1.79 \\
\hline 061126 & $0.06 \pm 0.04$ & $0.79 \pm 0.13$ & 14.23 & 0.73 & $0.06 \pm 0.04$ & $0.77 \pm 0.12$ & 13.87 & 0.63 & $0.07 \pm 0.04$ & $0.79 \pm 0.13$ & 14.16 & 0.47 \\
\hline 070125 & $0.15 \pm 0.02$ & $0.73 \pm 0.24$ & 13.30 & 6.53 & $0.14 \pm 0.04$ & $0.52 \pm 0.15$ & 10.11 & 0.56 & $0.17 \pm 0.04$ & $0.67 \pm 0.20$ & 12.47 & 1.86 \\
\hline 070306 & $1.41 \pm 0.25$ & $2.80 \pm 0.34$ & 14.45 & 1.89 & $1.34 \pm 0.29$ & $2.80 \pm 0.42$ & 15.23 & 1.96 & $1.34 \pm 0.30$ & $2.80 \pm 0.46$ & 16.02 & 1.90 \\
\hline 071003 & $0.13 \pm 0.08$ & $1.13 \pm 0.20$ & 18.13 & 0.79 & $0.10 \pm 0.05$ & $0.91 \pm 0.16$ & 14.82 & 0.69 & $0.24 \pm 0.05$ & $0.70 \pm 0.12$ & 11.78 & 0.51 \\
\hline 080319B & $0.10 \pm 0.08$ & $0.08 \pm 0.06$ & 4.24 & 2.35 & $0.14 \pm 0.05$ & $0.08 \pm 0.06$ & 4.24 & 1.32 & $0.08 \pm 0.06$ & $0.05 \pm 0.02$ & 4.24 & 1.67 \\
\hline 080330 & $0.23 \pm 0.16$ & $0.50 \pm 0.13$ & 10.00 & 3.03 & $0.25 \pm 0.11$ & $0.44 \pm 0.12$ & 9.19 & 3.19 & $0.28 \pm 0.14$ & $0.48 \pm 0.16$ & 9.70 & 3.23 \\
\hline 080514B & $0.10 \pm 0.04$ & $0.50 \pm 0.14$ & 8.60 & 0.50 & $0.08 \pm 0.05$ & $0.38 \pm 0.10$ & 6.89 & 0.59 & $0.08 \pm 0.05$ & $0.45 \pm 0.70$ & 7.86 & 0.43 \\
\hline 081008 & $0.20 \pm 0.10$ & $1.21 \pm 0.91$ & 20.52 & 5.56 & $0.38 \pm 0.28$ & $0.11 \pm 0.08$ & 4.15 & 1.44 & $0.16 \pm 0.12$ & $1.10 \pm 0.82$ & 18.86 & 3.83 \\
\hline
\end{tabular}

Note. The goodness of the fit is measured by $\chi^{2} / N_{\text {obs }}$, where $N_{\text {obs }}$ is the number of observational data points.

at slightly different epochs, we apply a correction using the temporal dependence of GRB afterglows $F_{v}(t) \propto t^{-\alpha}$ (where $\alpha$ is the decay index of the fitted light curve) and bring all values to the same epoch. To this end, we investigate the host galaxies of long-duration bursts for which a wealth of data on the afterglow are available. We build a sample composed of 33 long bursts. The sample is selected by requiring that (1) they are nearby or at a redshift $z<2$, and (2) high-quality multiband (mainly UV/optical/near-IR) afterglow photometry is available. This GRB host galaxy sample spans a redshift interval $0.125<z<1.98$, with a median value $z \simeq 1.1$. In Tables 1 and 2, we respectively tabulate the model parameters for fitting the afterglow SEDs using the MW, LMC, SMC extinction templates and the Drude approach. The fits are presented in Figures 1-11.

$G R B$ 970508. We constructed the afterglow SED $\left(I_{\mathrm{C}} R_{\mathrm{C}} V B U\right)$ of GRB 970508 at redshift $z \approx 0.835$ (Metzger et al. 1997a, $1997 b$ ), the second GRB for which an optical afterglow has been detected, with data from Galama et al. (1998) and Chary et al. (1998). The extinction in its host galaxy has been discussed by Reichart (1998), Wijers \& Galama (1999), Stratta et al. (2004), Kann et al. (2006), Starling et al. (2007), and others. Stratta et al. (2004) favored a MW-type extinction with $A_{V} \approx 0.27 \pm$ 0.16 mag. Kann et al. (2006) found $A_{V} \approx 0.38 \pm 0.11 \mathrm{mag}$ for the preferred MW-type extinction. Starling et al. (2007) argued against any significant extinction (and as a result they cannot distinguish between different extinction laws). In this work, the best-fit model is provided by a MW-type extinction of $A_{V} \approx 0.15 \pm 0.04$ mag but with a more prominent $2175 \AA$ bump (see Figure 1). If we neglect the $U$ band photometry, the Drude model would result in an extinction law similar to that of the SMC with $A_{V} \approx 0.49 \pm 0.11 \mathrm{mag}$.

$G R B$ 980703. We constructed the SED $\left(K H J I_{\mathrm{C}} R_{\mathrm{C}} V B\right)$ of GRB 980703 at $z \approx 0.966$ (Djorgovski et al. 1998) with data from Vreeswijk et al. (1999a) at $\sim 1.2$ days after the burst. Kann et al. (2006) derived $A_{V} \approx 1.32 \pm 0.59 \mathrm{mag}$ for the preferred SMC-type extinction. In this work, the Drude model results in $A_{V} \approx 1.25 \pm 0.45 \mathrm{mag}$ and an extinction law similar to that of the MW with $R_{V}=3.5$ (i.e., the extinction curve has a weaker $2175 \AA$ bump and flatter far-UV rise than that of the Galactic diffuse interstellar medium (ISM) of $R_{V} \approx 3.1$; see Cardelli et al. 1989) characteristics of denser regions (see Figure 1). The extinction appears to decrease with time (which was regarded as an indication of dust destruction by the burst; e.g., see Waxman \& Draine 2000; Draine \& Hao 2002; Perna et al. 2003): while Castro-Tirado et al. (1999) estimated $A_{V} \approx 2.2 \mathrm{mag}$ at $\sim 0.9$ days postburst, Vreeswijk et al. (1999a) estimated $A_{V} \approx 1.5 \pm 0.11 \mathrm{mag}$ at $\sim 1.2$ days and Bloom et al. (1998) 
Table 2

Parameters for Fitting the Afterglow SEDs with the "Drude" Approach

\begin{tabular}{|c|c|c|c|c|c|c|c|c|c|c|c|c|}
\hline GRB & $z$ & $\log \left(N_{\mathrm{H}} / \mathrm{cm}^{-2}\right)$ & Ref. & $c_{1}$ & $c_{2}$ & $c_{3}$ & $c_{4}$ & $A_{V}(\mathrm{mag})$ & $\beta$ & $R_{V}$ & $\log F_{\mathrm{o}}(\mu \mathrm{Jy})$ & $\chi^{2} / N_{\text {obs }}$ \\
\hline 970508 & 0.835 & $21.80 \pm 0.40$ & (1) & 0.24 & 1.32 & -2.00 & 0.12 & $0.15 \pm 0.04$ & $0.10 \pm 0.03$ & 3.14 & 43.56 & 0.20 \\
\hline 980703 & 0.966 & $22.56 \pm 0.20$ & (2) & 1.33 & 3.33 & -2.16 & 0.04 & $1.25 \pm 0.45$ & $1.19 \pm 0.42$ & 3.93 & 55.91 & 0.49 \\
\hline 990123 & 1.60 & $21.73 \pm 0.40$ & (1) & 1.60 & 2.90 & -2.00 & 0.00 & $0.30 \pm 0.06$ & $0.23 \pm 0.07$ & 3.25 & 20.77 & 0.30 \\
\hline 990510 & 1.619 & $22.00 \pm 0.30$ & (1) & 1.94 & 2.49 & -2.03 & 0.00 & $0.37 \pm 0.12$ & $0.28 \pm 0.10$ & 3.21 & 45.41 & 0.18 \\
\hline 991208 & 0.706 & $\ldots$ & $\cdots$ & 1.95 & 0.95 & -1.90 & 0.04 & $0.47 \pm 0.15$ & $0.48 \pm 0.12$ & 2.99 & 52.71 & 0.05 \\
\hline 991216 & 1.02 & $21.80 \pm 0.30$ & (3) & 0.06 & 0.49 & -2.00 & 0.06 & $0.14 \pm 0.05$ & $0.36 \pm 0.16$ & 3.12 & 44.04 & 0.16 \\
\hline 000911 & 1.058 & $\ldots$ & $\ldots$ & 0.62 & 2.62 & -2.02 & 0.06 & $0.32 \pm 0.10$ & $0.60 \pm 0.19$ & 3.09 & 51.68 & 0.23 \\
\hline 010222 & 1.477 & $22.08 \pm 0.20$ & (4) & 4.68 & 4.79 & -1.98 & 0.00 & $0.29 \pm 0.08$ & $0.81 \pm 0.19$ & 3.29 & 18.86 & 0.48 \\
\hline 010921 & 0.45 & $\ldots$ & $\cdots$ & 0.06 & 0.12 & -1.99 & 0.01 & $1.02 \pm 0.33$ & $0.52 \pm 0.17$ & 4.07 & 51.02 & 0.01 \\
\hline 011121 & 0.36 & $\ldots$ & $\ldots$ & 0.06 & 0.17 & -1.99 & 0.01 & $0.56 \pm 0.17$ & $0.49 \pm 0.14$ & 3.52 & 48.81 & 0.32 \\
\hline 020405 & 0.69 & $21.70 \pm 0.30$ & (5) & 0.21 & 0.74 & -1.96 & $1.0 \mathrm{E}-4$ & $0.72 \pm 0.14$ & $0.44 \pm 0.23$ & 3.26 & 21.20 & 0.90 \\
\hline 020813 & 1.25 & $20.88 \pm 0.12$ & (6) & 0.23 & 0.44 & -1.96 & 0.00 & $0.34 \pm 0.12$ & $0.46 \pm 0.09$ & 3.31 & 12.29 & 0.46 \\
\hline 030226 & 1.98 & $21.50 \pm 0.20$ & (7) & 0.52 & 0.88 & -1.96 & $1.0 \mathrm{E}-3$ & $0.24 \pm 0.04$ & $0.32 \pm 0.08$ & 3.20 & 28.97 & 0.36 \\
\hline 030328 & 1.52 & $20.78 \pm 0.18$ & (8) & 0.22 & 1.28 & -2.00 & 0.00 & $0.20 \pm 0.06$ & $0.32 \pm 0.12$ & 3.26 & 44.89 & 0.27 \\
\hline 030329 & 0.168 & $20.30 \pm 0.05$ & (9) & 0.02 & 0.28 & -2.00 & 0.00 & $0.30 \pm 0.12$ & $0.60 \pm 0.23$ & 3.29 & 35.37 & 0.18 \\
\hline 040924 & 0.859 & $\ldots$ & $\ldots$ & 0.22 & 1.26 & -1.94 & 0.00 & $0.36 \pm 0.10$ & $0.40 \pm 0.14$ & 3.26 & 19.64 & 0.56 \\
\hline 041006 & 0.716 & $21.51 \pm 0.02$ & (8) & 0.55 & 1.00 & -2.00 & 0.00 & $0.15 \pm 0.04$ & $0.32 \pm 0.12$ & 3.19 & 37.82 & 0.20 \\
\hline 050318 & 1.44 & $\ldots$ & $\cdots$ & 1.59 & 1.20 & -2.00 & 0.00 & $1.51 \pm 0.54$ & $1.17 \pm 0.42$ & 3.04 & 40.00 & 0.17 \\
\hline 050408 & 1.2357 & $22.08 \pm 0.12$ & (10) & 1.51 & 1.11 & -2.17 & 0.03 & $0.47 \pm 0.08$ & $0.62 \pm 0.10$ & 3.00 & 55.56 & 0.30 \\
\hline $050525 \mathrm{~A}$ & 0.606 & $21.34 \pm 0.09$ & (11) & 1.22 & 0.85 & -1.61 & 0.04 & $0.85 \pm 0.23$ & $0.63 \pm 0.18$ & 3.24 & 47.50 & 0.02 \\
\hline 050824X & 0.83 & $\ldots$ & $\ldots$ & 1.45 & 0.92 & -1.67 & 0.05 & $0.23 \pm 0.07$ & $0.21 \pm 0.04$ & 3.16 & 45.52 & 0.13 \\
\hline 051111 & 1.55 & $21.90 \pm 0.15$ & (12) & 0.61 & 0.50 & -1.91 & 0.03 & $0.38 \pm 0.10$ & $0.37 \pm 0.10$ & 3.37 & 24.34 & 0.28 \\
\hline 060614 & 0.125 & $20.48 \pm 0.12$ & (13) & 0.30 & 2.86 & -1.89 & 0.00 & $0.42 \pm 0.18$ & $0.46 \pm 0.22$ & 3.29 & 22.94 & 0.35 \\
\hline 060729 & 0.54 & $21.30 \pm 0.08$ & (14) & 0.50 & 0.91 & -2.00 & 0.00 & $0.45 \pm 0.26$ & $0.49 \pm 0.17$ & 3.20 & 42.94 & 0.30 \\
\hline 061121 & 1.314 & $21.91 \pm 0.10$ & (15) & 8.45 & 5.34 & -0.99 & 0.10 & $1.10 \pm 0.44$ & $0.56 \pm 0.23$ & 3.29 & 51.52 & 1.39 \\
\hline 061126 & 1.1588 & $21.43 \pm 0.05$ & (16) & 0.17 & 1.16 & -2.00 & 0.00 & $0.03 \pm 0.01$ & $0.90 \pm 0.15$ & 3.07 & 41.53 & 0.20 \\
\hline 070125 & 1.547 & $\ldots$ & $\ldots$ & 0.14 & 0.21 & -1.95 & $2.0 \mathrm{E}-3$ & $0.45 \pm 0.13$ & $0.22 \pm 0.06$ & 3.99 & 36.78 & 0.25 \\
\hline 070306 & 1.4959 & $21.59 \pm 0.04$ & (17) & 0.50 & 0.32 & -1.98 & 0.00 & $1.40 \pm 0.32$ & $2.60 \pm 0.43$ & 3.69 & 20.34 & 1.87 \\
\hline 071003 & 1.60435 & $\ldots$ & $\ldots$ & 0.22 & 1.08 & -2.01 & 0.02 & $0.51 \pm 0.14$ & $0.48 \pm 0.08$ & 3.26 & 37.21 & 0.47 \\
\hline 080319B & 0.937 & $21.27 \pm 0.03$ & (18) & 1.36 & 0.66 & -1.97 & 0.00 & $0.05 \pm 0.02$ & $0.12 \pm 0.04$ & 3.06 & 52.42 & 0.48 \\
\hline 080330 & 1.51 & $\ldots$ & $\ldots$ & 0.24 & 1.30 & -1.99 & $2.0 \mathrm{E}-3$ & $0.41 \pm 0.12$ & $0.28 \pm 0.07$ & 3.26 & 14.73 & 2.19 \\
\hline 080514B & 1.8 & $21.15 \pm 0.30$ & (19) & 0.30 & 1.68 & -2.10 & 0.01 & $0.24 \pm 0.06$ & $0.32 \pm 0.13$ & 3.26 & 42.19 & 0.29 \\
\hline 081008 & 1.9685 & $21.83 \pm 0.05$ & (20) & 2.24 & 1.80 & -1.99 & $1.0 \mathrm{E}-3$ & $0.31 \pm 0.23$ & $0.48 \pm 0.36$ & 3.08 & 50.05 & 1.20 \\
\hline
\end{tabular}

References. (1) Galama \& Wijers 2001; (2) Vreeswijk et al. 1999a; (3) Ballantyne et al. 2002; (4) Stratta et al. 2004; (5) Mirabal et al. 2003; (6) Butler et al. 2003; (7) Klose et al. 2004; (8) Butler et al. 2005; (9) Hjorth et al. 2003; (10) Foley et al. 2006; (11) Blustin et al. 2006; (12) Butler et al. 2006; (13) Dado et al. 2008; (14) Grupe et al. 2007; (15) Page et al. 2007; (16) Gomboc et al. 2008; (17) Jaunsen et al. 2008; (18) Bloom et al. 2009; (19) Rossi et al. 2008; (20) Racusin et al. 2008.

derived $A_{V} \approx 0.9 \pm 0.2 \mathrm{mag}$ at $\sim 5.3$ days, all assuming a MWtype extinction law. However, using the SMC extinction as a template, Starling (2008) examined this burst in detail and did not find any evidence of variable extinction.

$G R B$ 990123. We constructed the SED $\left(K H I_{\mathrm{C}} R_{\mathrm{C}} V B U\right)$ of GRB 990123 at $z \approx 1.60$ (Akerlof et al. 1999) with data from Galama et al. (1999). Kann et al. (2006) argued for a SMC-type extinction law with very little extinction $A_{V} \approx 0.04 \pm 0.05 \mathrm{mag}$, while Savaglio et al. (2003) estimated $A_{V} \approx 1.1$ mag from metal column abundances. We found $A_{V} \approx 0.30 \pm 0.06$ mag with an extinction law similar to that of the MW but lacking the $2175 \AA$ extinction bump (see Figure 1). ${ }^{8}$

$G R B$ 990510. We constructed the SED $\left(I_{\mathrm{C}} R_{\mathrm{C}} V B\right)$ of GRB 990510 at $z \approx 1.619$ (Vreeswijk et al. 1999b) with data from Stanek et al. (1999) and Beuermann et al. (1999). Stanek et al. (1999) suggested that the deviation of the $B$-band flux from

\footnotetext{
8 While it is true that the extinction curve derived here from the Drude model deviates from the MW extinction law at $\lambda^{-1}>9 \mu \mathrm{m}^{-1}$, we note that the uncertainty of the far-UV part of the MW extinction curve (i.e., the observed dispersion of the curve) is not small (e.g., at $\lambda^{-1}=10 \mu \mathrm{m}^{-1}$ the uncertainty exceeds 30\%; see Figure 1 of Zubko et al. 2004). The Galactic average extinction curve is based on the Astronomical Netherlands Satellite work by Savage et al. (1985) covering about 1000 sightlines. On the other hand, the extinction curve of the GRB host at $\lambda^{-1}>9 \mu \mathrm{m}^{-1}$ would indeed be better constrained if the afterglow data at $\lambda^{-1}>9 \mu \mathrm{m}^{-1}$ are available.
}

the $F_{v} \propto v^{-0.46 \pm 0.08}$ power law fitted to the $V R_{\mathrm{C}} I_{\mathrm{C}}$ fluxes may indicate an extinction of $A_{V} \approx 0.45 \mathrm{mag}$ of the host galaxy or an intervening galaxy at $z \approx 1.62$. Kann et al. (2006) derived $A_{V} \approx 0.18 \pm 0.24 \mathrm{mag}$ and a SMC-type extinction law. The Drude model also suggests a featureless extinction curve (with $A_{V} \approx 0.37 \pm 0.12 \mathrm{mag}$ ) like that of the SMC, but not as steep (see Figure 2).

$G R B$ 991208. We constructed the $\operatorname{SED}\left(K I_{\mathrm{C}} R_{\mathrm{C}} V B\right)$ of GRB 991208 at $z \approx 0.706$ (Dodonov et al. 1999; Djorgovski et al. 1999) with data from Castro-Tirado et al. (2001) and Chary et al. (2002). Kann et al. (2006) found that the MW-type extinction with $A_{V} \approx 0.80 \pm 0.29$ mag provides a better fit to the observed afterglow SED than the LMC and SMC models. The Drude approach derives an extinction law with a weak $2175 \AA$ bump and a far-UV rise even steeper than that of the SMC (see Figure 2), similar to the extinction curve of the high latitude translucent cloud toward HD 210121 (Larson et al. 1996; Li \& Greenberg 1998), which has the steepest far-UV rise in the Galaxy. This model requires $A_{V} \approx 0.47 \pm 0.15 \mathrm{mag}$.

$G R B$ 991216. We constructed the SED $\left(K H J I_{\mathrm{C}} R_{\mathrm{C}} V B\right)$ of GRB 991216 at $z \approx 1.02$ (Vreeswijk et al. 1999c) with data from Garnavich et al. (2000) and Halpern et al. (2000). The Drude approach derives $A_{V} \approx 0.14 \pm 0.05 \mathrm{mag}$ and an extinction curve very similar to that of the MW (see Figure 2). The presence 

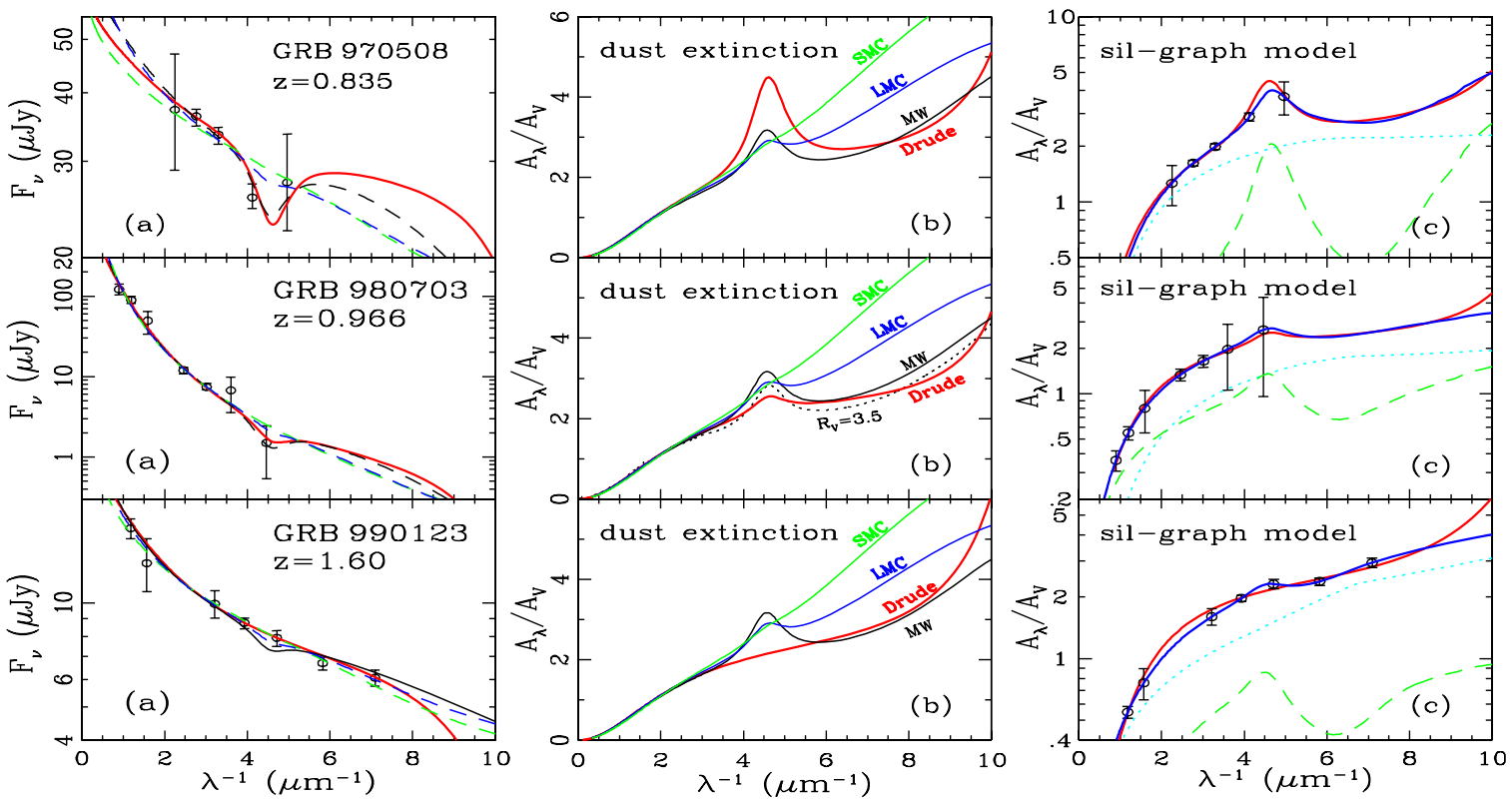

Figure 1. Upper panel (a): fitting the SED of the afterglow of GRB 970508 with the "Drude" approach (red) and the MW (black), LMC (blue), and SMC (green) templates for the GRB host extinction curve. Upper panel (b): comparison of the MW (black), LMC (blue), and SMC (green) extinction laws with that derived from the Drude approach (red). Upper panel (c): fitting the derived extinction curve (red solid line and black filled circles) with a mixture of amorphous silicate (cyan dotted line) and graphite dust (green dashed line). The blue solid line plots the resulting model extinction curve. Middle panel: same as the upper panel but for GRB 980703. Also shown in the middle panel (b) is the MW extinction curve of $R_{V}=3.5$ (dashed line). Bottom panel: same as the upper panel but for GRB 990123.

(A color version of this figure is available in the online journal.)
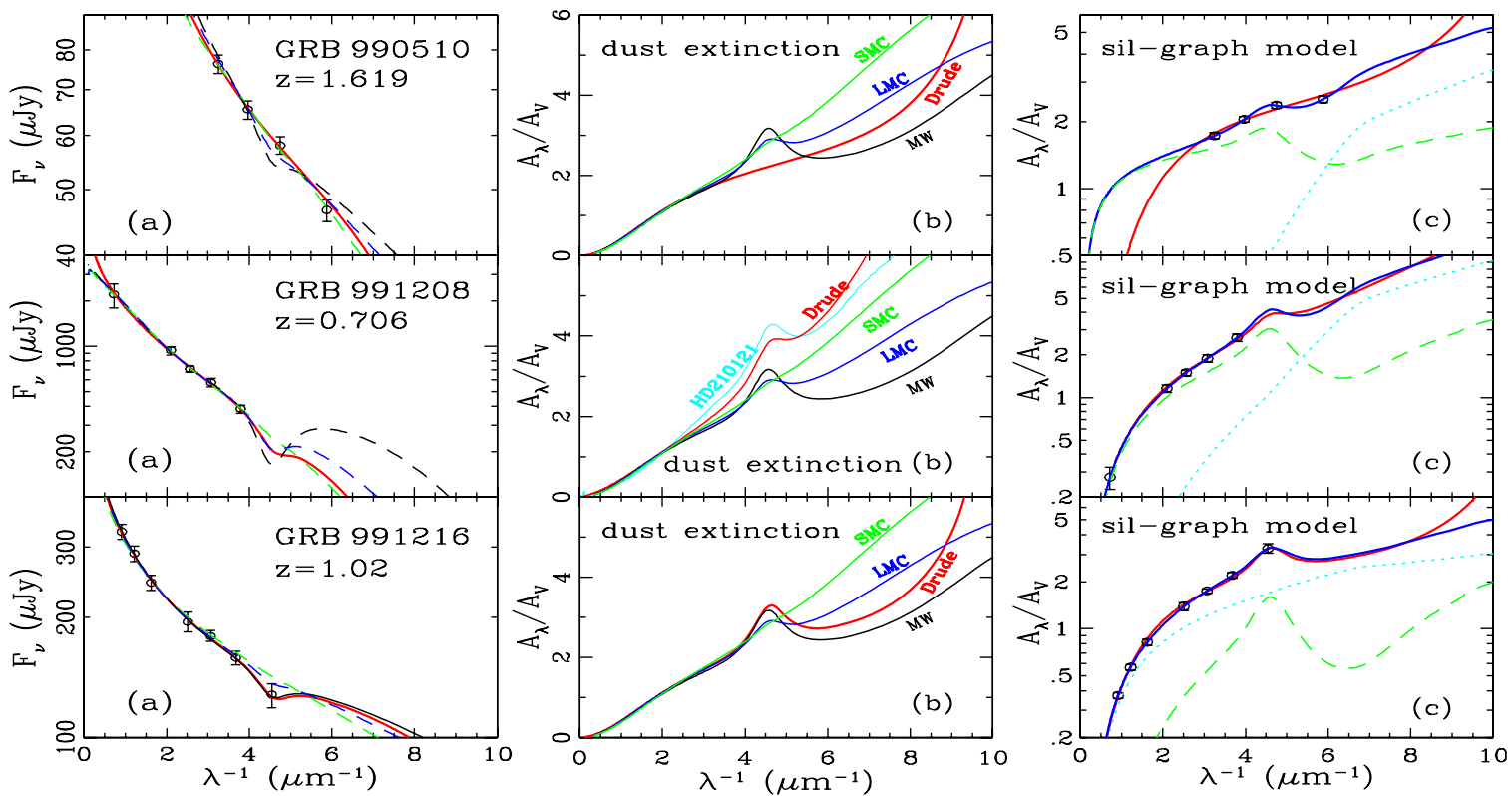

Figure 2. Same as Figure 1 but for GRB 990510, GRB 991208, and GRB 991216. Also shown in the middle panel (b) is the extinction curve of the high latitude translucent cloud toward HD 210121 (thin cyan line) which has the steepest far-UV rise ever observed in the Galaxy.

(A color version of this figure is available in the online journal.)

of the $2175 \AA$ bump in its extinction curve was supported by the detection of the broad absorption feature centered at $\sim 2360 \AA$ (Vreeswijk et al. 2006). Kann et al. (2006) also found that the MW-type extinction (with $A_{V} \approx 0.13 \pm 0.08 \mathrm{mag}$ ) is preferred.

$G R B$ 000911. We constructed the SED $\left(K_{\mathrm{S}} J I_{\mathrm{C}} R_{\mathrm{C}} V B\right)$ of GRB 000911 at $z \approx 1.058$ (Price et al. 2002a) with data from Lazzati et al. (2001) and Price et al. (2002a). Masetti et al. (2005) found a SMC-type extinction of $A_{V} \approx 0.32 \mathrm{mag}$, while Kann et al. (2006) found the MW-type extinction of $A_{V} \approx 0.27 \pm 0.32 \mathrm{mag}$ provides a slightly better fit than the LMC and SMC models. The Drude approach results in $A_{V} \approx 0.32 \pm 0.10 \mathrm{mag}$ and an extinction curve very similar to that of the MW (see Figure 3). If we ignore the $B$-band photometry, a SMC-type extinction would be preferred.

$G R B$ 010222. We constructed the SED $\left(K J I_{\mathrm{C}} R_{\mathrm{C}} V B U\right)$ of GRB 010222 at $z \approx 1.477$ (Jha et al. 2001) with data from Masetti et al. (2001). The Drude approach derives $A_{V} \approx$ $0.29 \pm 0.08 \mathrm{mag}$ with an extinction curve similar to that of the MW but with the $2175 \AA$ bump removed (see Figure 3). In literature, both low and high $A_{V}$ values were suggested: 

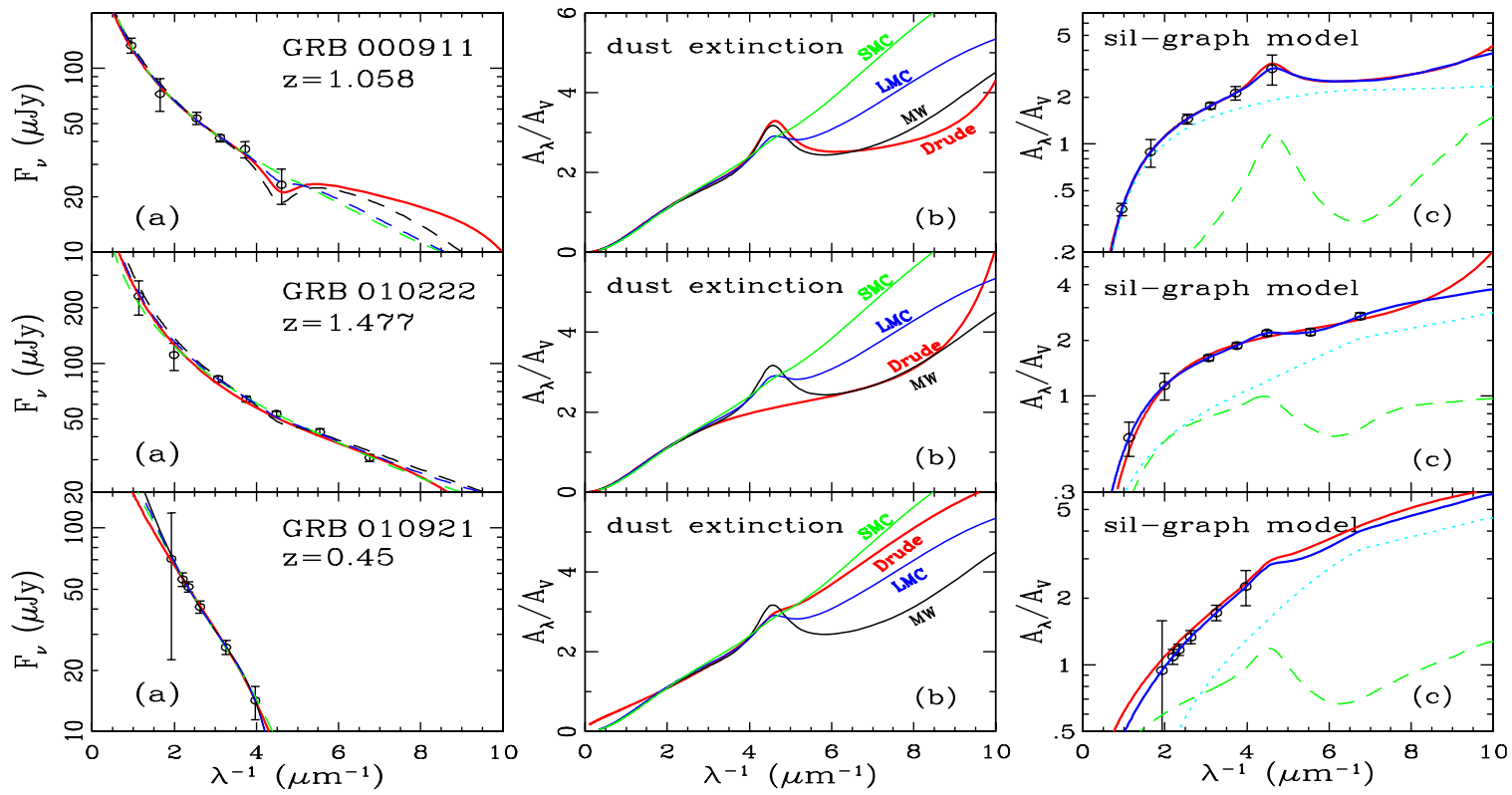

Figure 3. Same as Figure 1 but for GRB 000911, GRB 010222, and GRB 010921.

(A color version of this figure is available in the online journal.)
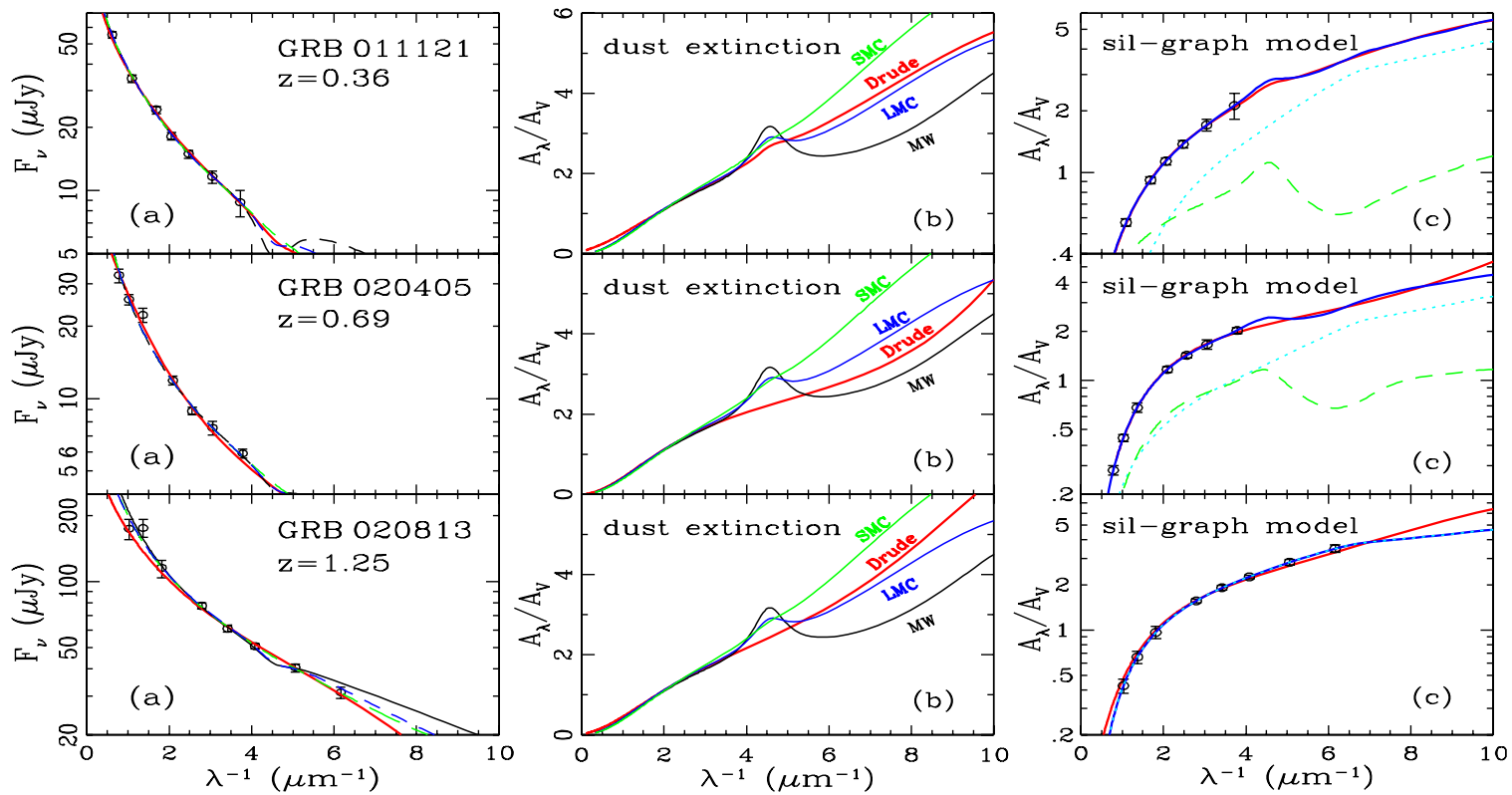

Figure 4. Same as Figure 1 but for GRB 011121, GRB 020405, and GRB 020813.

(A color version of this figure is available in the online journal.)

(1) using the extinction curve model of Reichart (2001), ${ }^{9}$ Lee et al. (2001) found $A_{V}<0.06 \mathrm{mag}$ (with $\beta=0.75$ fixed) and Galama et al. (2003) found $A_{V} \approx 0.11 \pm 0.02 \mathrm{mag}$; (2) Kann et al. (2006) were in favor of a SMC-type extinction with $A_{V} \approx 0.14 \pm 0.08 \mathrm{mag}$; and (3) Savaglio et al. (2003) argued

\footnotetext{
9 The seven-parameter analytical formula proposed by Reichart (2001) for the extinction curve $A_{\lambda} / A_{V}$ of GRB hosts was based on the expressions of Cardelli et al. (1989) for $\lambda>0.3 \mu \mathrm{m}$ and of Fitzpatrick \& Massa (1990) for $0.1 \mu \mathrm{m}<\lambda<0.3 \mu \mathrm{m}$. The problem with the Reichart (2001) formula (see his Equations (61) and (66)) is that, as mentioned earlier, the CCM expression is only valid for the Galactic extinction curves, it is not suitable for the SMC or LMC extinctions (Gordon et al. 2003). Therefore, if a GRB host happens to have a SMC- or a LMC-type extinction law, models based on the Reichart (2001) formula will not be able to restore the true extinction (while the Drude approach does).
}

for a much higher extinction of $A_{V} \approx 0.7$ mag from a study based on the depletions of the dust-forming elements $\mathrm{Fe}$ and $\mathrm{Si}$.

$G R B$ 010921. We constructed the SED $\left(i^{*} r^{*} R_{\mathrm{C}} V B U\right)$ of GRB 010921 at $z \approx 0.45$ (Bloom et al. 2001) with data from Price et al. (2002b). Kann et al. (2006) preferred a MW-type extinction with $A_{V} \approx 0.81 \pm 1.21 \mathrm{mag}$. Price et al. (2003) inferred a LMC-type extinction of $A_{V} \approx 1 \mathrm{mag}$. The Drude approach results in an extinction curve very similar to that of the SMC (see Figure 3 ) with $A_{V} \approx 1.02 \pm 0.33 \mathrm{mag}$.

$G R B$ 011121. We constructed the SED $\left(K J I_{\mathrm{C}} R_{\mathrm{C}} V B U\right)$ of GRB 011121 at $z \approx 0.36$ (Infante et al. 2001) with data from Garnavich et al. (2003) and Greiner et al. (2003c). While Kann et al. (2006) preferred a SMC-type extinction with $A_{V}=0.39 \pm 0.14 \mathrm{mag}$, the Drude approach derives 

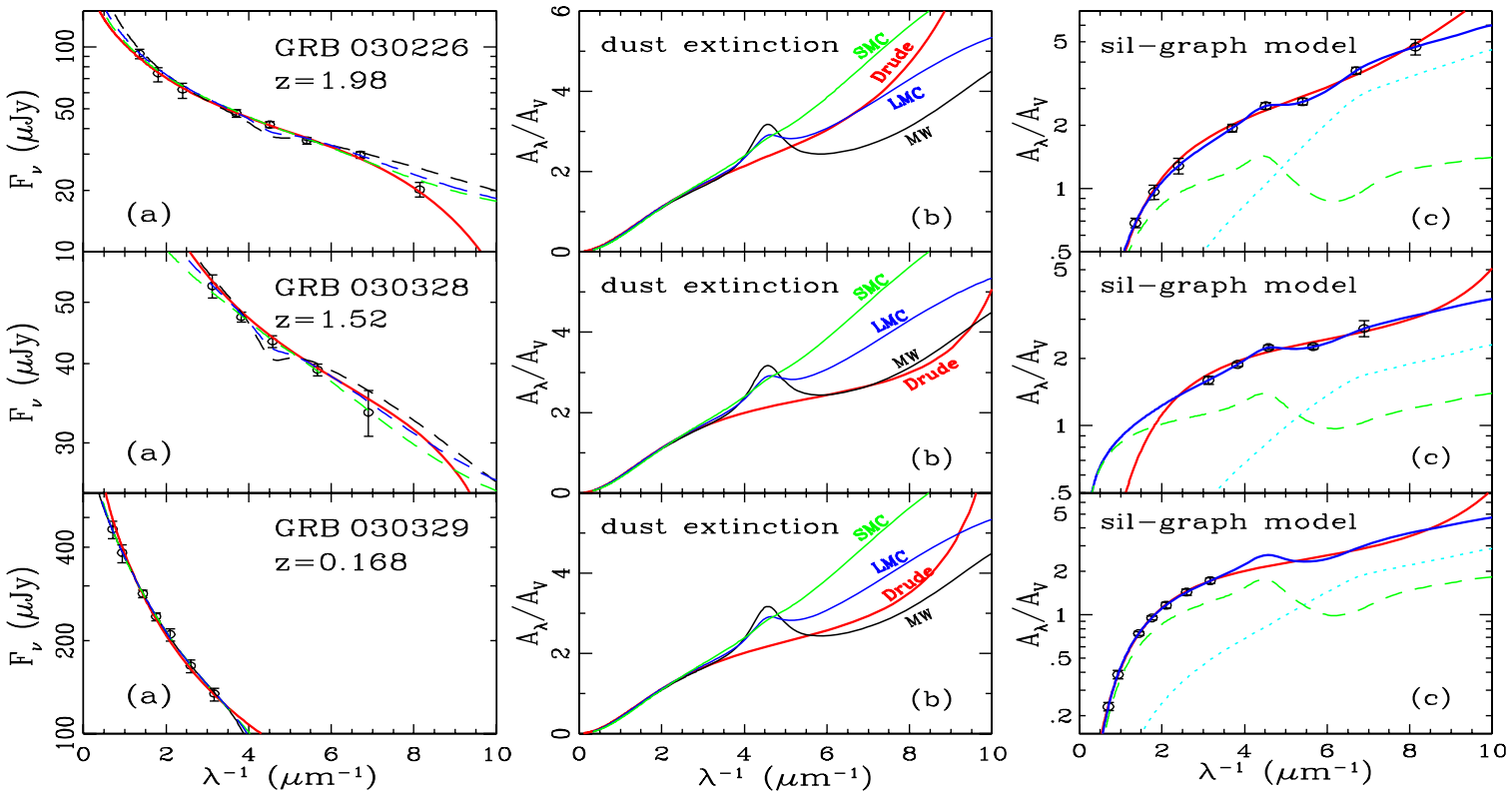

Figure 5. Same as Figure 1 but for GRB 030226, GRB 030328, and GRB 030329.

(A color version of this figure is available in the online journal.)
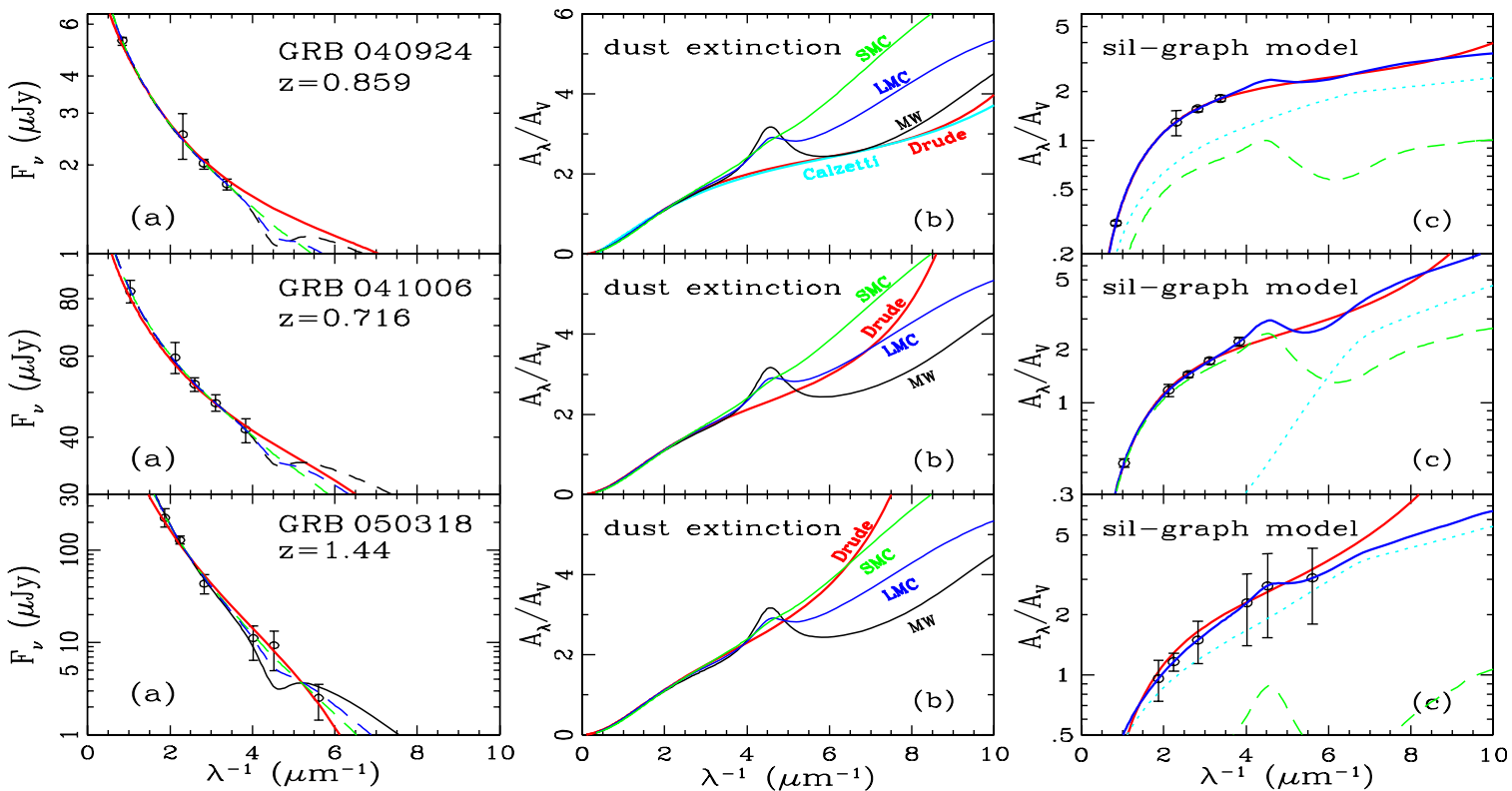

Figure 6. Same as Figure 1 but for GRB 040924, GRB 041006, and GRB 050318. Also shown in the upper panel (b) is the so-called "Calzetti" attenuation law of starburst galaxies (cyan line)

(A color version of this figure is available in the online journal.)

$A_{V} \approx 0.56 \pm 0.17 \mathrm{mag}$ and an extinction curve very similar to that of the LMC but with a much weaker $2175 \AA$ bump (see Figure 4). ${ }^{10}$

$G R B$ 020405. We constructed the SED $\left(K_{\mathrm{S}} H J I_{\mathrm{C}} R_{\mathrm{C}} V B\right)$ of GRB 020405 at $z \approx 0.69$ (Masetti et al. 2002) with data from Masetti et al. (2003). While Stratta et al. (2005) argued for a flat, gray extinction curve with $A_{V} \approx 2.3 \mathrm{mag}$ based on a comparison of the X-ray and the optical data, Kann et al. (2006) found a moderate extinction of $A_{V} \approx 0.25 \mathrm{mag}$ for the SMC, LMC, and MW template extinction laws. The Drude approach

\footnotetext{
${ }^{10}$ The $c_{4}$ parameter in Equation (2) which measures the strength of the $2175 \AA$ bump is only $\approx 0.01$ for GRB 011121 , while $c_{4} \approx 0.022,0.052$ for the LMC and MW extinction laws, respectively.
}

infers a featureless extinction curve steeply rising toward the far-UV with $A_{V} \approx 0.72 \pm 0.14 \mathrm{mag}$ (see Figure 4 ).

$G R B$ 020813. We constructed the SED $\left(K H J I_{\mathrm{C}} R_{\mathrm{C}} V B U\right)$ of GRB 020813 at $z \approx 1.25$ (Price et al. 2002c) with data from Barth et al. (2003). The Drude approach infers an extinction law just like that of the LMC but with the $2175 \AA$ bump removed (see Figure 4). The inferred extinction $A_{V} \approx 0.34 \pm 0.12 \mathrm{mag}$ is higher than that derived by Kann et al. (2006; $A_{V} \approx$ $0.12 \pm 0.07 \mathrm{mag}$ for SMC-type extinction), by Covino et al. (2003; $A_{V} \approx 0.12 \pm 0.04 \mathrm{mag}$ for MW-type extinction), and by Savaglio \& Fall (2004; $A_{V}<0.19 \mathrm{mag}$ from an analysis of the metal column densities).

$G R B$ 030226. We constructed the SED $\left(K H J I_{\mathrm{C}} R_{\mathrm{C}} V B\right)$ of GRB 030226 at $z \approx 1.98$ (Greiner et al. 2003a) with data 

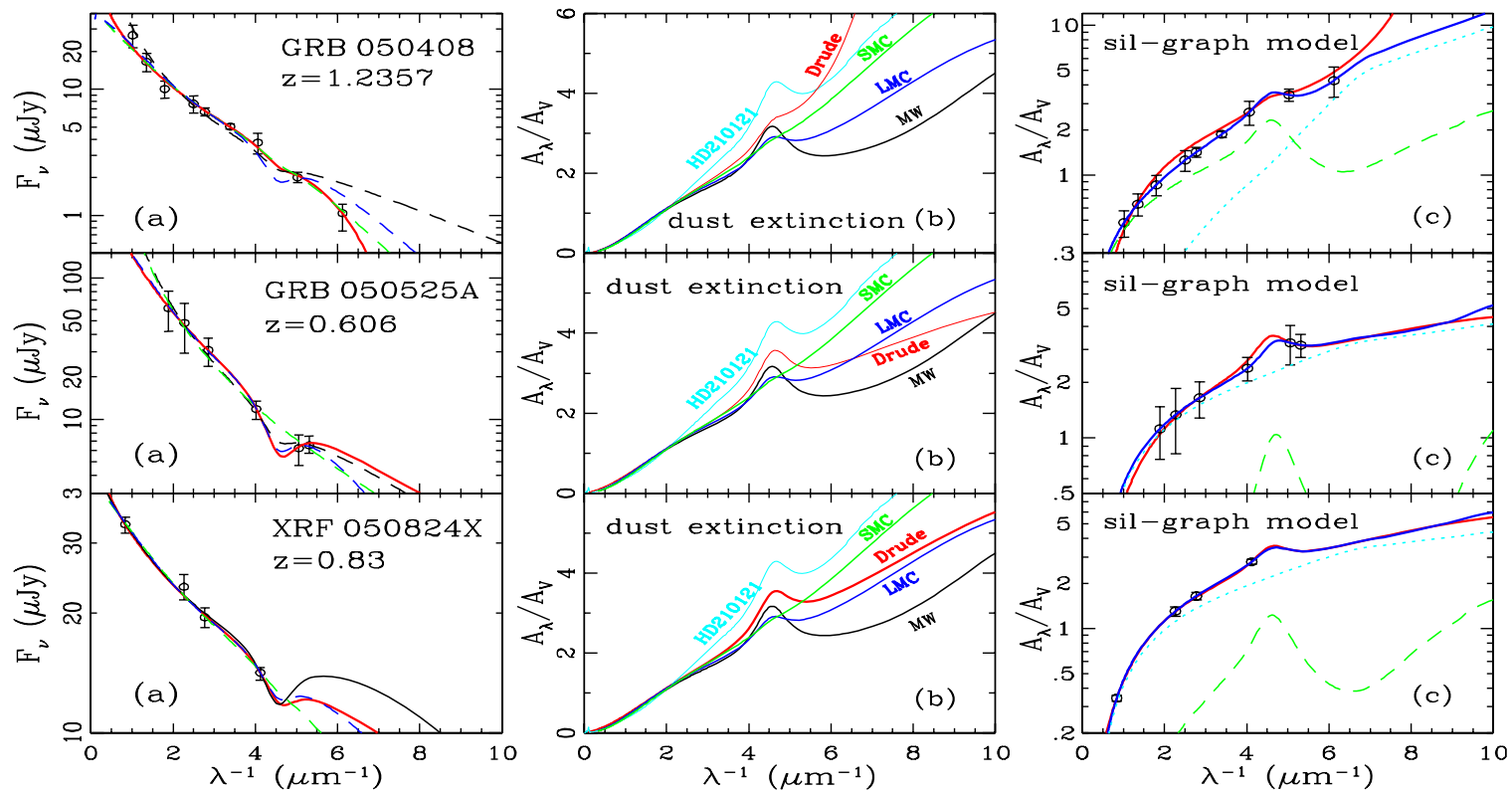

Figure 7. Same as Figure 1 but for GRB 050408, GRB 050525A, and XRF 050824X. Also shown in the upper panel (b), the middle panel (b), and the bottom panel (b) is the extinction curve of the high latitude translucent cloud toward HD 210121 (thin cyan line), which has the steepest far-UV rise ever observed in the Galaxy.

(A color version of this figure is available in the online journal.)
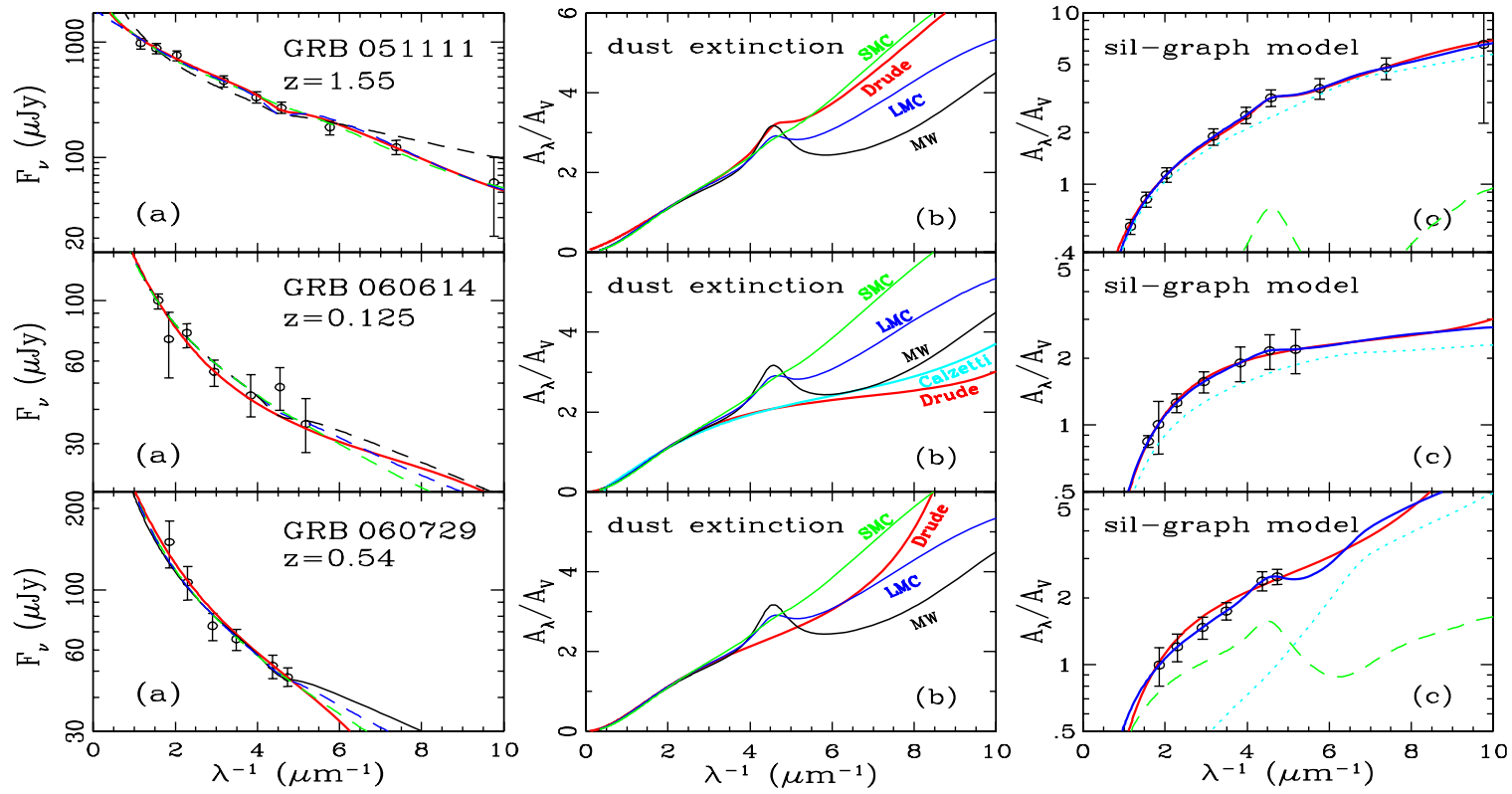

Figure 8. Same as Figure 1 but for GRB 051111, GRB 060614, and GRB 060729. Also shown in the middle panel (b) is the "Calzetti" law (cyan line).

(A color version of this figure is available in the online journal.)

from Klose et al. (2004). Kann et al. (2006) found negligible extinction ( $A_{V} \approx 0.06 \pm 0.06 \mathrm{mag}$ for SMC-type extinction), in consistent with Klose et al. (2004). The Drude approach infers $A_{V} \approx 0.24 \pm 0.04 \mathrm{mag}$ and an extinction law resembling that of the LMC but with the $2175 \AA$ bump removed and a steeper far-UV rise at $\lambda^{-1}>8 \mu \mathrm{m}^{-1}$ (see Figure 5).

$G R B$ 030328. We constructed the SED $\left(I_{\mathrm{C}} R_{\mathrm{C}} V B U\right)$ of GRB 030328 at $z \approx 1.52$ (Martini et al. 2003) with data from Maiorano et al. (2006). From the Fe II column density derived from the VLT optical spectrum of its afterglow, Maiorano et al. (2006) estimated $A_{V}<0.1 \mathrm{mag}$. Kann et al. (2006) fitted the afterglow SED with a SMC-type extinction of $A_{V} \approx 0.05 \pm 0.15 \mathrm{mag}$. The Drude approach derives
$A_{V} \approx 0.20 \pm 0.06 \mathrm{mag}$ with an extinction curve just like that of the MW but with the $2175 \AA$ bump removed (see Figure 5).

$G R B$ 030329. We constructed the SED $\left(H J I_{\mathrm{C}} R_{\mathrm{C}} V B U\right)$ of GRB 030329 at $z \approx 0.168$ (Greiner et al. 2003b) with data from Gorosabel et al. (2005). Bloom et al. (2004) found $A_{V} \approx$ $0.30 \pm 0.03 \mathrm{mag}$ (with $\beta=0.5$ fixed; $A_{V} \approx 0.94 \pm 0.24 \mathrm{mag}$ if $\beta$ was treated as a free parameter) assuming a MW-type extinction. Kann et al. (2006) derived $A_{V} \approx 0.54 \pm 0.22 \mathrm{mag}$ $(\beta \approx 0.30 \pm 0.22)$ with a SMC-type extinction. The Drude approach results in $A_{V} \approx 0.30 \pm 0.12 \mathrm{mag}(\beta \approx 0.60 \pm 0.23)$ with a featureless extinction curve which is steeper than the MW curve but not as steep as that of the LMC and the SMC (see Figure 5). 

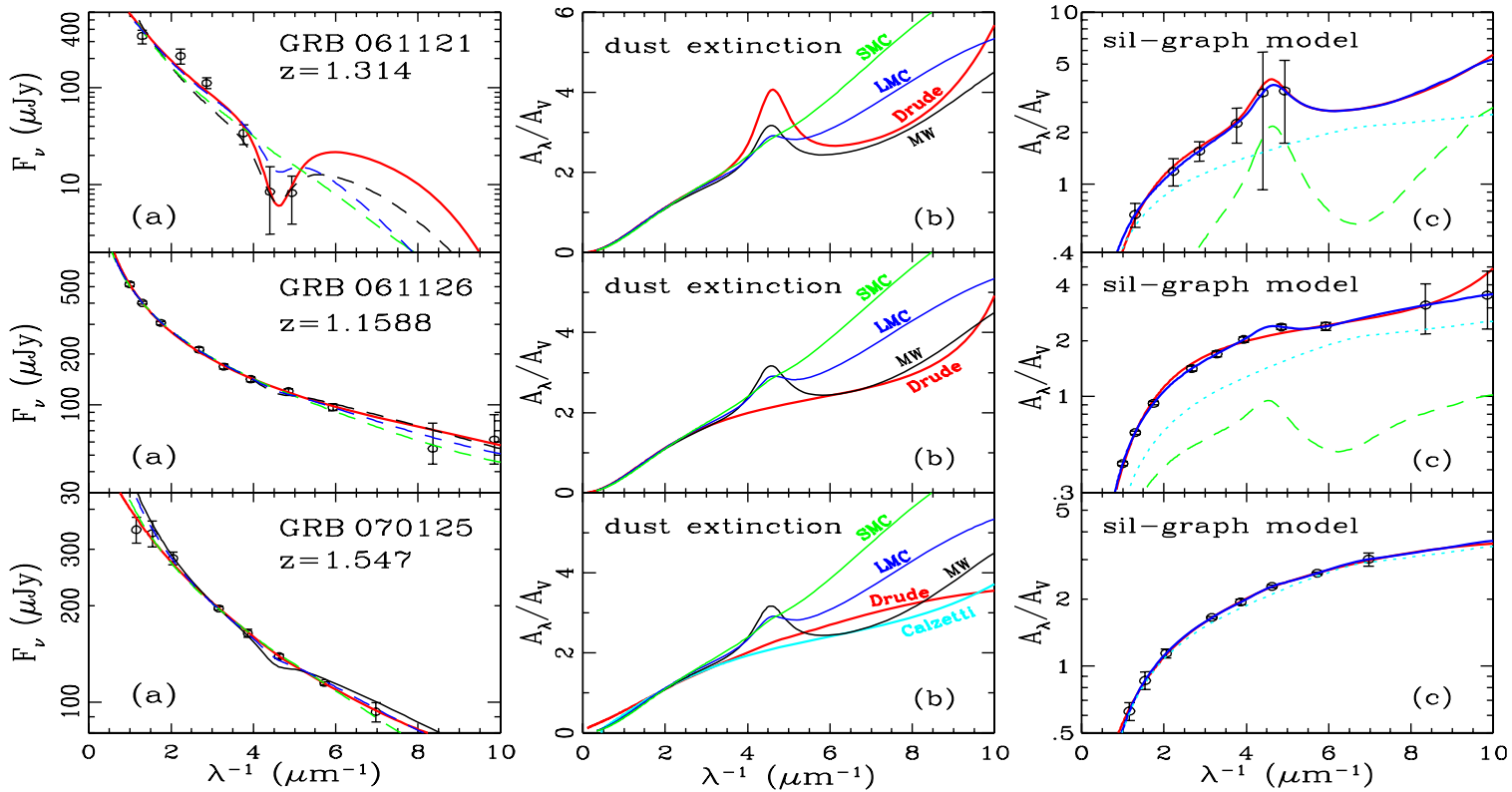

Figure 9. Same as Figure 1 but for GRB 061121, GRB 061126, and GRB 070125. Also shown in the bottom panel (b) is the "Calzetti" law (cyan line).

(A color version of this figure is available in the online journal.)
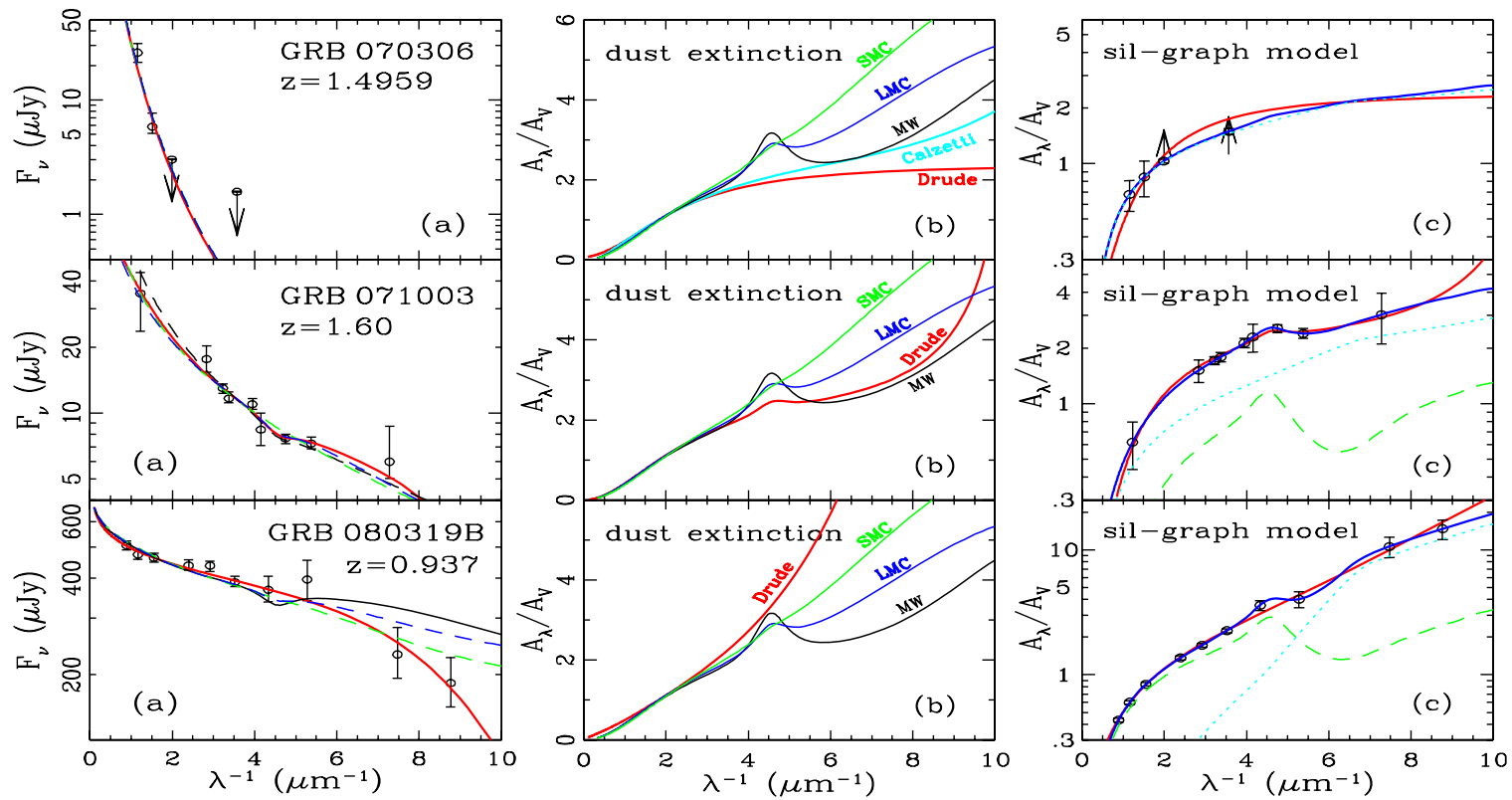

Figure 10. Same as Figure 1 but for GRB 070306, GRB 071003, and GRB 080319B. Also shown in the upper panel (b) is the "Calzetti" law (cyan line).

(A color version of this figure is available in the online journal.)

$G R B$ 040924. We constructed the SED $\left(K I_{\mathrm{C}} R_{\mathrm{C}} V\right)$ of GRB 040924 at $z \approx 0.859$ (Wiersema et al. 2004) with data from Silvey et al. (2004). Due to the lack of data at wavelengths bluer than the $V$ band, Kann et al. (2006) found that the MW, LMC, and SMC extinction laws all provide excellent fits to the afterglow SED with $A_{V} \approx 0.21 \pm 0.62 \mathrm{mag}$ and $\beta \approx 0.59 \pm 0.61$ (MW), $A_{V} \approx 0.22 \pm 0.62 \mathrm{mag}$ and $\beta \approx 0.58 \pm 0.64$ (LMC), and $A_{V} \approx 0.16 \pm 0.44 \mathrm{mag}$ and $\beta \approx 0.63 \pm 0.48$ (SMC). The Drude approach yields a featureless, relatively flat extinction curve with $A_{V} \approx 0.36 \pm 0.10 \mathrm{mag}$ and $\beta \approx 0.40 \pm 0.14$ (see Figure 6). The derived extinction curve is almost identical to that of the so-called "Calzetti" attenuation law of starburst galaxies. This also provides another piece of evidence for the advantage of the Drude approach: if the "true" extinction curve of a
GRB host happens to resemble the "Calzetti" law which is also widely used as a template, the "Drude" approach will allow us to restore it.

$G R B$ 041006. We constructed the SED $\left(H I_{\mathrm{C}} R_{\mathrm{C}} V B\right)$ of GRB 041006 at $z \approx 0.716$ (Price et al. 2004) with data from Soderberg et al. (2006). As shown in Figure 6, both the Drude approach and the approach assuming a template extinction law (MW, LMC, and SMC) closely fit the afterglow SED (as due to the lack of data at wavelengths bluer than the $B$ band). The Drude approach infers an extinction curve similar to that of the LMC but with a steeper far-UV rise at $\lambda>7.5 \mu \mathrm{m}^{-1}$ and without the $2175 \AA$ bump. This model yields $A_{V} \approx 0.15 \pm 0.04 \mathrm{mag}$ and $\beta \approx 0.32 \pm 0.12$, in comparison with that of Kann et al. (2006): $A_{V} \approx 0.11 \pm 0.23 \mathrm{mag}$ and $\beta \approx 0.36 \pm 0.27$ (MW), 

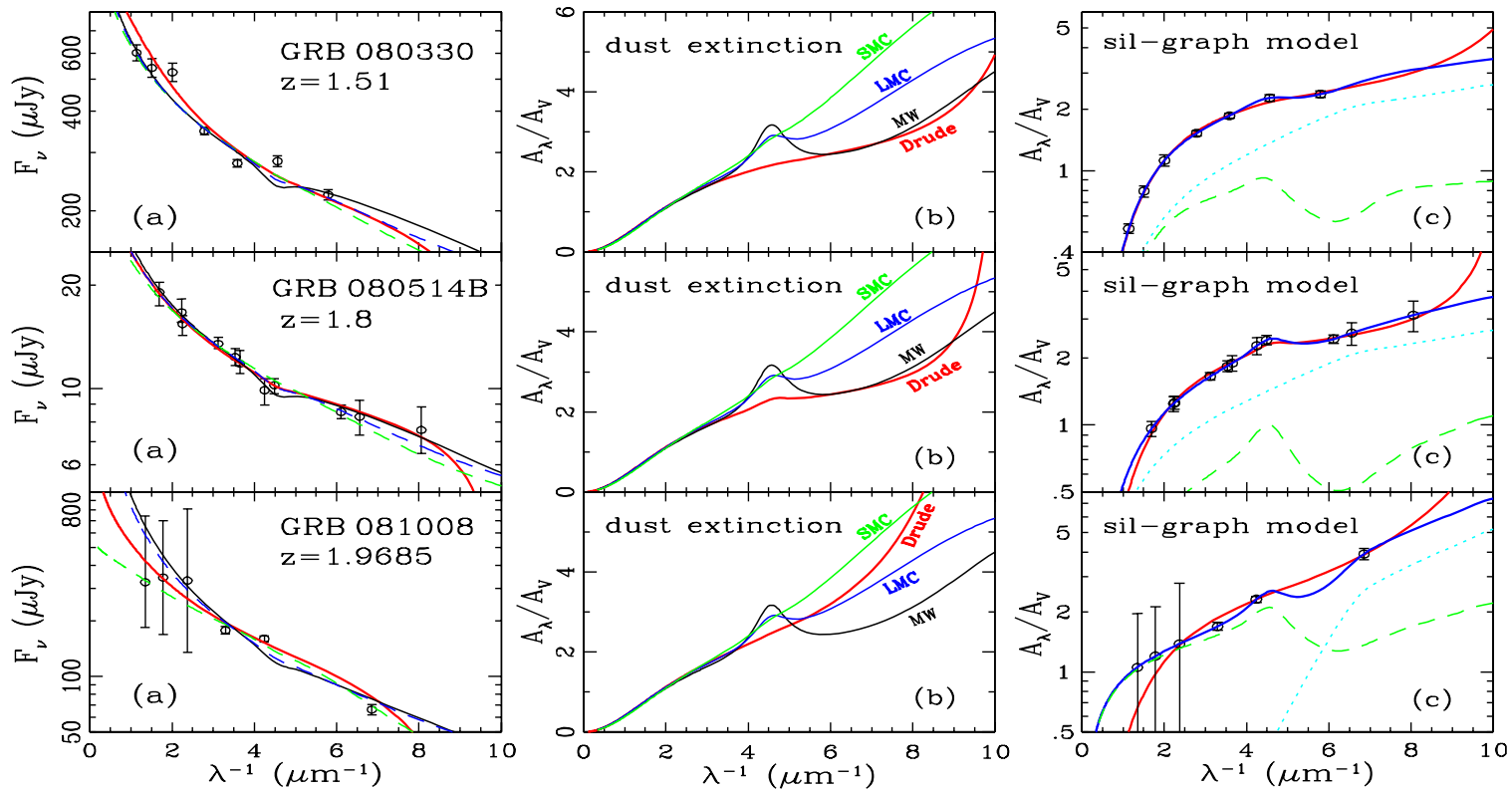

Figure 11. Same as Figure 1 but for GRB 080330, GRB 080514B, and GRB 081008.

(A color version of this figure is available in the online journal.)

$A_{V} \approx 0.14 \pm 0.28 \mathrm{mag}$ and $\beta \approx 0.32 \pm 0.33$ (LMC), and $A_{V} \approx 0.12 \pm 0.23 \mathrm{mag}$ and $\beta \approx 0.34 \pm 0.30$ (SMC).

$G R B$ 050318. We constructed the SED (VBU, UVW1, UVM2, UVW2) of GRB 050318 at $z \approx 1.44$ (Berger \& Mulchaey 2005) with data from Still et al. (2005) and Perri et al. (2005). Perri et al. (2005) and Still et al. (2005) both derived $\beta \approx$ $1.1 \pm 0.1$. The Drude approach yields $A_{V} \approx 1.51 \pm 0.54 \mathrm{mag}$ and $\beta \approx 1.17 \pm 0.42$, with an extinction curve similar to that of the SMC (see Figure 6).

GRB 050408. We constructed the SED (KHJIRVBU) of GRB 050408 at $z \approx 1.2357$ (Prochaska et al. 2005) with data from Wiersema et al. (2005), Milne et al. (2005), Kahharov et al. (2005), Flasher et al. (2005a), Foley et al. (2006), and de Ugarte Postigo et al. (2007). The Drude approach yields $A_{V} \approx 0.47 \pm 0.08 \mathrm{mag}$ (and $\beta \approx 0.62 \pm 0.10$ ) and an extinction curve even steeper than that of the SMC and exhibiting a weak $2175 \AA$ bump, as probably revealed by the curvature in the $U B V$ fluxes (see Figure 7). In contrast, de Ugarte Postigo et al. (2007) and Kann et al. (2007) were in favor of a SMC-type extinction with $A_{V} \approx 0.74 \pm 0.15 \mathrm{mag}$ and $\beta \approx 0.28 \pm 0.33$. The extinction curve derived here is steeper than that of the MW with $R_{V}=2.5$ but not as steep as that of HD 210121.

$G R B$ 050525A. We constructed the SED (VBU, UVW1, UVM2, UVW2) of GRB 050525A at $z \approx 0.606$ (Foley et al. 2005) with data from Cobb \& Bailyn (2005), Yanagisawa et al. (2005), Kaplan et al. (2005), Flasher et al. (2005b), Klotz et al. (2005), Blustin et al. (2006), and Della Valle et al. (2006a). The Drude approach yields $A_{V} \approx 0.85 \pm 0.23 \mathrm{mag}$ and $\beta \approx 0.63 \pm 0.18$ at $\sim 200 \mathrm{~s}$ postburst and an extinction curve lying in between that of HD 210121 and that of the MW with $R_{V}=2.5$ (see Figure 7). The derived extinction is much higher than that of Schady et al. (2007; $A_{V} \approx 0.16 \pm 0.03 \mathrm{mag}$ ), Kann et al. (2007; $A_{V} \approx 0.32 \pm 0.20 \mathrm{mag}$ ), and Blustin et al. (2006; $\left.A_{V} \approx 0.23 \pm 0.15 \mathrm{mag}\right)$.

$X R F$ 050824X. We constructed the SED (KIRB) of $\mathrm{XRF} 050824 \mathrm{X}$ at $z \approx 0.83$ (Fynbo et al. 2005) with data from Schady et al. (2007a) and Sollerman et al. (2007). The Drude approach deduces $A_{V} \approx 0.23 \pm 0.07 \mathrm{mag}$ and $\beta \approx 0.21 \pm 0.04$ with an extinction curve lying in between that of HD 210121 and that of the MW with $R_{V}=2.5$ (see Figure 7), similar to GRB 050525A. Assuming a SMC-type extinction, Kann et al. (2007) found $A_{V} \approx 0.14 \pm 0.13 \mathrm{mag}$ and $\beta \approx 0.45 \pm 0.18$ (but Sollerman et al. 2007 derived $A_{V} \approx 0.4 \pm 0.2 \mathrm{mag}$ and $\beta \approx 0.56 \pm 0.04$ ). We note that the limited wavelength coverage of these data does not really allow one to discern the SMC, LMC, MW, and Drude extinction.

GRB 051111. We constructed the SED ( $K_{\mathrm{S}} H J I R V B U$, UVW1, UVM2, UVW2) of GRB 051111 at $z \approx 1.55$ (Hill et al. 2005) with data from Bloom (2005) and Yost et al. (2007). Butler et al. (2006) and Kann et al. (2007) were both in favor of a SMC-type extinction, with $A_{V} \approx 0.23 \pm 0.07 \mathrm{mag}$ and $A_{V} \approx 0.19 \pm 0.02 \mathrm{mag}$, respectively. The Drude approach deduces $A_{V} \approx 0.38 \pm 0.10 \mathrm{mag}$ and $\beta \approx 0.37 \pm 0.10$ with an extinction curve resembling that of the SMC but with a very weak $2175 \AA$ bump (see Figure 8). If we ignore the curvature of the $R V B$ photometry, we would obtain a SMC-type extinction law.

$G R B$ 060614. We constructed the SED (RVBU, UVW1, UVM2, UVW2) of GRB 060614 at $z \approx 0.125$ (Price et al. 2006) with data from Della Valle et al. (2006b) and Mangano et al. (2007). While Mangano et al. (2007) derived $A_{V} \approx 0.05 \mathrm{mag}$ with a SMC-like extinction law, the Drude approach infers $A_{V} \approx 0.42 \pm 0.18 \mathrm{mag}$ and $\beta \approx 0.46 \pm 0.22$ with a featureless, relatively flat extinction curve, similar to that of the "Calzetti" law (see Figure 8).

GRB 060729. We constructed the SED (VBU, UVW1, UVM2, UVW2) of GRB 060729 at $z \approx 0.54$ (Thoene et al. 2006) with data from Grupe et al. (2006, 2007). The Drude approach fits the afterglow SED at $\sim 100$ ks postburst with an extinction curve resembling that of the LMC but with a steeper far-UV rise at $\lambda^{-1}>7 \mu \mathrm{m}^{-1}$ (see Figure 8). This model requires $A_{V} \approx 0.45 \pm 0.26 \mathrm{mag}$ (and $\beta \approx 0.49 \pm 0.17$ ).

$G R B$ 061121. We constructed the SED $\left(R i^{\prime} V B U\right.$, UVW1, UVM2, UVW2) of GRB 061121 at $z \approx 1.314$ (Bloom et al. 2006) with data from Yost et al. (2006), Melandri et al. (2006), and Page et al. (2007). The Drude approach infers 
$A_{V} \approx 1.10 \pm 0.44$ mag with an extinction curve resembling that of the MW but with a stronger $2175 \AA$ A bump (see Figure 9). ${ }^{11}$ The MW-type extinction model fits the $2175 \AA$ bump region very well, but fails at $\lambda^{-1}<3.7 \mu \mathrm{m}^{-1}$. If we ignore both the $U$ and $B$ bands, we would then prefer a SMC-type extinction curve.

GRB 061126. We constructed the SED (KHJIRVBU, UVW1, UVM2) of GRB 061126 at $z \approx 1.1588$ with data from Perley et al. (2008a). With a priori assumption of template extinction laws with a fixed wavelength dependence, Kann et al. (2007) found that the SMC-type extinction provides the best fit with $A_{V} \approx 0.095 \pm 0.055 \mathrm{mag}$, while Perley et al. (2008a) argued that a strong gray extinction may be responsible for the difference between the faint optical subluminosity and the bright X-ray afterglow of this burst. The Drude approach infers $A_{V} \approx 0.03 \pm 0.01 \mathrm{mag}$ with an extinction curve just like that of the MW but with the $2175 \AA$ bump removed (see Figure 9).

GRB 070125. We constructed the SED $\left(K_{S} J H I R V B U\right)$ of GRB 070125 at $z \approx 1.547$ (Fox et al. 2007) with data from Updike et al. (2008). Due to the lack of the $2175 \AA$ A bump, Updike et al. (2008) argued for a SMC-type extinction with $A_{V} \approx$ $0.11 \mathrm{mag}$. The Drude approach infers $A_{V} \approx 0.45 \pm 0.13 \mathrm{mag}$ with a featureless, relatively flat extinction curve resembling that of the "Calzetti" law (see Figure 9).

GRB 070306. We constructed the afterglow SED (RJHK) of GRB 070306 at $z \approx 1.4959$ (Jaunsen et al. 2008) with data from Jaunsen et al. (2008). Jaunsen et al. (2008) explored the overall extinction of the host galaxy by reddening a starburst galaxy template SED (Kinney et al. 1996) with various extinction laws. They found the upper limit of $A_{V}$ in the range $\sim 0.1-$ $0.5 \mathrm{mag}$, with the best fit given by a MW-type extinction ( $A_{V} \approx 0.17 \mathrm{mag}$ ). However, the afterglow of this burst seems to be highly extinguished: while it was not detected in the optical wavelengths, a near-IR afterglow was clearly detected. Jaunsen et al. (2008) fitted the $H K$ fluxes and the $R J$ upper limits of the afterglow of this burst, by requiring the intrinsic spectral slope of the near-IR afterglow $\beta_{\mathrm{o}}$ to relate to that of the X-ray afterglow $\beta_{\mathrm{x}}$ through $\beta_{\mathrm{o}}=\beta_{\mathrm{x}}-0.5$ (i.e., assuming a broken power law with the cooling break occurring between the near-IR and X-ray bands). They found $A_{V} \approx 5.5 \pm 0.6 \mathrm{mag}$ and a SMC-type extinction. The Drude approach infers $A_{V} \approx 1.40 \pm 0.32 \mathrm{mag}$ (and $\beta \approx 2.60 \pm 0.43$ ) and a flat extinction law (see Figure 10). The major reason why we obtain a much lower $A_{V}$ is that our $\beta$ value is higher than that of Jaunsen et al. (2008) who fixed it to be $\beta=0.7$. We do not want to overinterpret our results since we only have four data points among which two of them are just upper limits. We will investigate this burst in a separate paper, with special attention paid to the "true" $\beta$ value.

GRB 071003. We constructed the SED (ugVrRiIz) of GRB 071003 at $z \approx 1.60435$ with data from Perley et al. (2008b). While Perley et al. (2008b) found a SMC-type extinction and $A_{V} \approx 0.21 \mathrm{mag}$, the Drude approach infers $A_{V} \approx 0.51 \pm 0.14 \mathrm{mag}$ and an extinction law resembling that of the MW but with a much weaker $2175 \AA$ bump and a steeper far-UV rise at $\lambda^{-1}>8.5 \mu \mathrm{m}^{-1}$ (see Figure 10).

GRB 080319B. We constructed the SED (KHJIRVBU, UVW1, UVM2, UVW2) of GRB 080319B at $z \approx 0.937$ (Vreeswijk et al. 2008) with data from Bloom et al. (2009). While Bloom et al. (2009) were in favor of a SMC-type extinction law with $A_{V} \approx 0.07 \pm 0.02 \mathrm{mag}$, the Drude approach

\footnotetext{
11 The $c_{4}$ parameter of Equation (2) which measures the strength of the $2175 \AA$ bump is $\approx 0.10$ for GRB 061121 , while $c_{4} \approx 0.052$ for the MW extinction law.
}

deduces $A_{V} \approx 0.05 \mathrm{mag}$ and a featureless extinction law even steeper than that of the SMC (see Figure 10).

GRB 080330. We constructed the SED (KHJIRVB) of GRB 080330 at $z \approx 1.51$ (Malesani et al. 2008) with data from Cobb (2008a). The Drude approach derives $A_{V} \approx 0.41 \pm$ 0.12 mag and an extinction law closely resembling that of the MW but lacking the $2175 \AA$ Aump (see Figure 11).

GRB 080514B. We constructed the SED (NEWFIRM $J$, GROND $J$, GROND $z^{\prime}, I_{\mathrm{C}}$, Gemini $i$, GROND $i, R_{\mathrm{C}}$, GROND $r^{\prime}$, GROND $g^{\prime}, B$, and $U$ ) of GRB 080514B at $z \approx 1.80$ (Rossi et al. 2008) with data from Rossi et al. (2008). While Rossi et al. (2008) found no evidence for dust in the host galaxy, the Drude approach infers $A_{V} \approx 0.24 \pm 0.06 \mathrm{mag}$ and an extinction law resembling that of the MW but with a much weaker $2175 \AA$ \& bump and a steeper far-UV rise at $\lambda^{-1}>9 \mu \mathrm{m}^{-1}$ (see Figure 11).

GRB 081008. We constructed the SED (KHJIRB) of GRB 081008 at $z \approx 1.9685$ (D'Avanzo et al. 2008) with data from Cobb (2008b). The Drude approach infers $A_{V} \approx$ $0.31 \pm 0.23 \mathrm{mag}$ and an extinction law resembling that of the LMC but lacking the $2175 \AA$ bump and with a steeper far-UV rise at $\lambda^{-1}>6.5 \mu \mathrm{m}^{-1}$ (see Figure 11).

\section{DISCUSSION}

Unlike the models based on a pre-assumption of a specific extinction law (which as a result restricts the derived extinction laws to one of the adopted templates), the Drude approach is more flexible in determining the true wavelength dependence of the host extinction. As shown in Section 3, the Drude approach reveals a wide diversity of extinction laws for the sample of 33 GRBs.

1. Some bursts exhibit an extinction law more or less like that of the MW $\left(R_{V} \approx 3.1\right.$ for GRB 991216 and 000911, and $R_{V}>3.5$ for GRB 980703), LMC (GRB 011121 but with a weaker $2175 \AA$ A bump), and SMC (GRB 010921, 050318 and 051111) - this demonstrates that if the extinction law of a GRB host happens to resemble one of the widely adopted template extinction laws (e.g., the MW, LMC, and SMC laws), the Drude approach is able to restore the templatelike true extinction law.

2. Some bursts exhibit an extinction law just like that of the MW (with $R_{V} \approx 3.1$ ) but (1) with a stronger $2175 \AA$ bump (GRB 970508 and 061121), (2) with a much weaker $2175 \AA$ bump (GRB 071003 and 080514B), or (3) simply with the $2175 \AA$ bump completely removed (GRB 990123, 010222, 030328, 061126, and 080330).

3. Some bursts exhibit an extinction law like that of the high Galactic latitude translucent cloud HD 210121 (Larson et al. 1996; Li \& Greenberg 1998) or lying in between that of HD 210121 and that of the MW with $R_{V}=2.5$, which is characteristics of a weak $2175 \AA$ bump and a steep far-UV rise (GRB 991208, 050408, 050525A, and XRF 050824X).

4. Some bursts exhibit a featureless extinction law which steeply rises toward the far-UV (GRB 990510, 020405, 020813, 030226, 030329, 041006, 060729, 080319B, and 081008 ), with a varying degree of steepness.

5. Some bursts exhibit a featureless, flat extinction law (GRB 040924, 060614, 070125, and 070306). Some of them are similar to the so-called "Calzetti" attenuation law of starburst galaxies (GRB 040924, 060614, and 070125). 


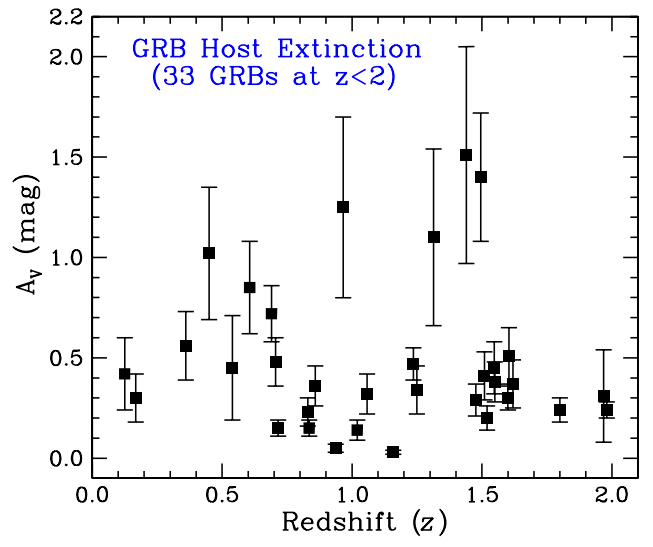

Figure 12. Derived host galaxy visual extinction $A_{V}$ (in the source frame) as a function of redshift $z$. For the 33 bursts at $z<2$ discussed in this work, we do not see any strong evidence for the dependence of $A_{V}$ on $z$.

(A color version of this figure is available in the online journal.)

The extinction quantities (see Figure 12), the shapes of the deduced extinction curves, and the presence or absence of the $2175 \AA$ bump do not seem to correlate with redshifts (more details will be presented in S. L. Liang \& A. Li 2010, in preparation).

For bursts lacking the $2175 \AA$ A bump, models based on a priori assumption of specific template extinction laws often infer a SMC-type extinction with a small $A_{V}$. As demonstrated in this work, among the 19 GRBs (of the entire sample of 33 bursts) which lack the $2175 \AA$ A bump, only three bursts (GRB 010921, 050318, and 051111) have an extinction curve similar to that of the SMC. The extinction curves of the other 16 bursts display a wide range of shapes, ranging from being relatively flat to very steep. The $A_{V}$ values derived from the Drude approach is generally $\sim 2-5$ times larger than that derived from assuming a SMC template extinction law provided that the "true" extinction law inferred from the Drude approach is not as steep as that of the SMC.

In Figure 13(a), we plot the distribution of $A_{V}$. We see that the distribution of $A_{V}$ peaks at $\sim 0.4 \mathrm{mag}$, with a strong clustering toward relative low extinction $\left(A_{V}<0.6 \mathrm{mag}\right)$, which is higher than that of Kann et al. (2006; $A_{V}<0.2 \mathrm{mag}$ ) who were in favor of a SMC-type extinction. This also indicates that if the "true" extinction law of a GRB host galaxy is not like that of the SMC, one would underestimate $A_{V}$ by taking the SMC extinction law to be the template. We should note that the distribution of $A_{V}$ inferred here may not reflect the "true" extinction distribution as the sample selected here is biased to bursts with bright optical afterglows and high-quality photometry data (so highly extinguished bursts like dark bursts are unlikely included in this sample).

In Figure 13(b), we show the distribution of $R_{V}$ calculated from the deduced extinction curves. We see that most of these bursts have $R_{V}$ in the range of $\sim 3.0-3$.4. But the actual extinction curves differ substantially from predicted from the CCM formula using these $R_{V}$ values (needless to mention the absence of the $2175 \AA$ bump of which the presence is predicted from the CCM formula). Even the very steep extinction curve of GRB 080319B (see Figure 10) has $R_{V} \approx 3.06$. This is not surprising since the CCM formula is known to be valid only for the Galactic sightlines (e.g., see Gordon et al. 2003).

We select a sample of 25 GRBs having known hydrogen column densities $N_{\mathrm{H}}$ which range from $1.0 \times 10^{19} \mathrm{~cm}^{-2}$ to $1.0 \times 10^{23} \mathrm{~cm}^{-2}$ (see Kann et al. 2006 for references). In
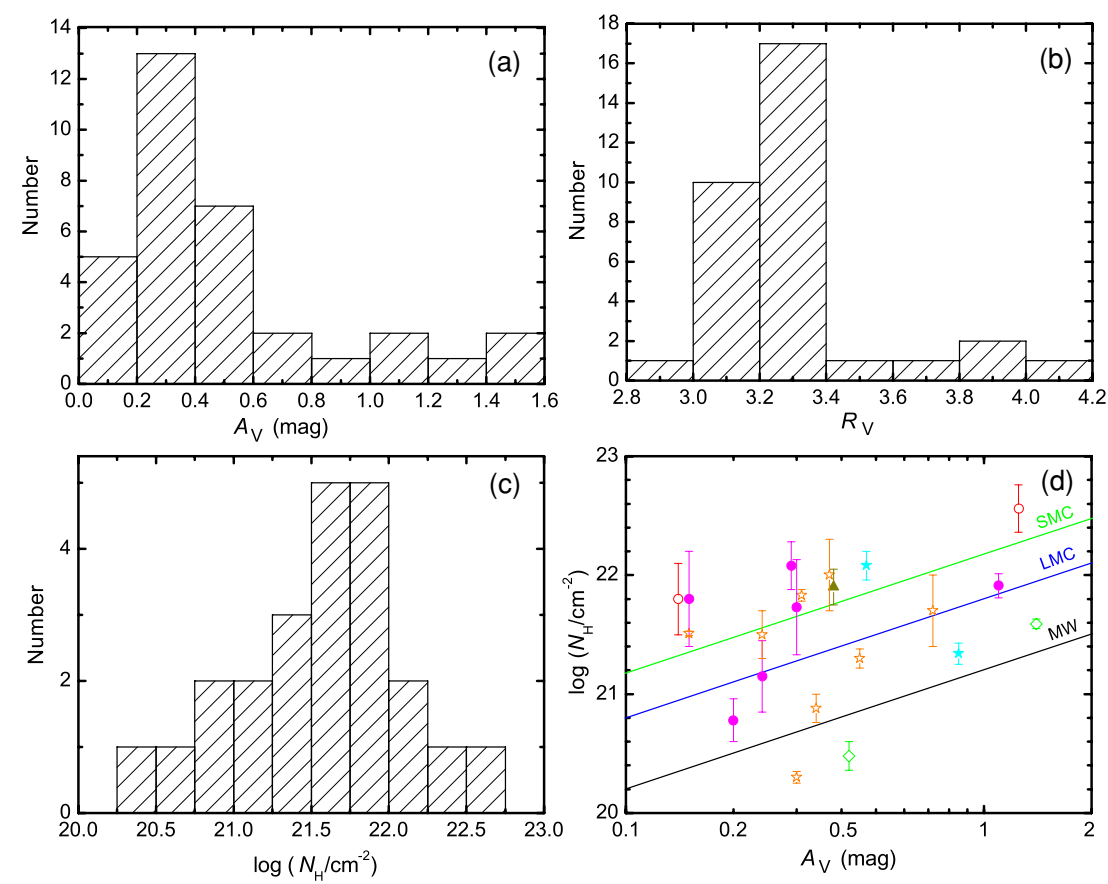

Figure 13. (a) Distribution of the derived host galaxy visual extinction $A_{V}$. (b) Distribution of the total-to-selective extinction ratio $R_{V} \equiv A_{V} / E(B-V)$. (c) Distribution of the hydrogen column densities $N_{\mathrm{H}}$ along the lines of sight toward the bursts in their host galaxies. (d) Dust-to-gas ratios in the host galaxies along the lines of sight toward 25 GRBs. Also plotted are that of the MW, LMC, and SMC. Open circles: those bursts with a MW-type extinction; open triangles: those with a LMC-type extinction; filled triangles: those with a SMC-type extinction; filled circles: those with a MW-type extinction but with a very weak (or lacking) $2175 \AA$ bump; open stars: those with a featureless, steep extinction curve; filled stars: those with a steep far-UV rise and a weak $2175 \AA$ bump; open diamonds: those with a flat curve.

(A color version of this figure is available in the online journal.) 


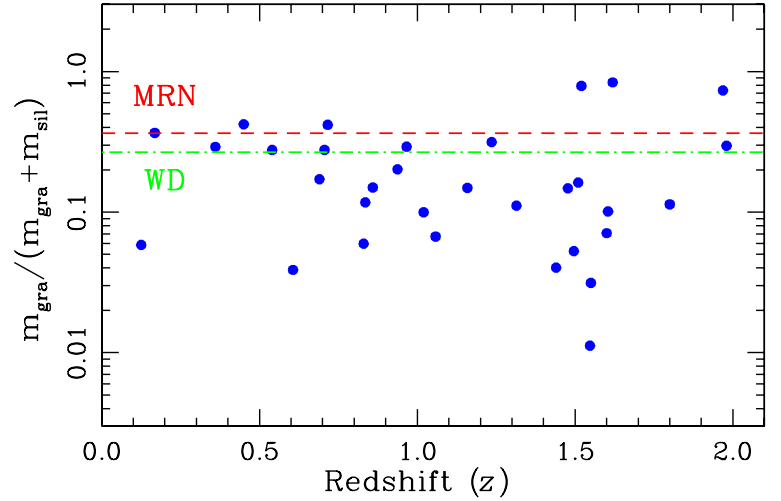

Figure 14. Mass fractions of graphite dust as a function of redshift $z$ for all 33 bursts at $z<2$. Also shown are the mass fractions of graphite of the MRN silicate-graphite model (red dashed line; Mathis et al. 1977) and the WD silicate-graphite-polycyclic aromatic hydrocarbon model (green dot-dashed line; Weingartner \& Draine 2001).

(A color version of this figure is available in the online journal.)

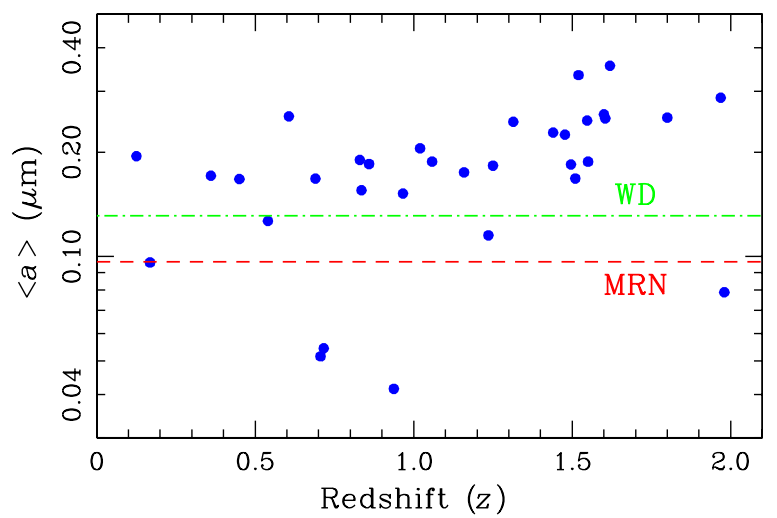

Figure 15. Mass-weighted mean dust sizes as a function of redshift $z$. Also shown are the mean dust sizes of the MRN silicate-graphite model (red dashed line; Mathis et al. 1977) and the WD silicate-graphite-polycyclic aromatic hydrocarbon model (green dot-dashed line; Weingartner \& Draine 2001).

(A color version of this figure is available in the online journal.)

Figure 13(c), we show the distribution of $N_{\mathrm{H}}$ for these bursts. We see that the majority of these bursts have $N_{\mathrm{H}}$ in the range of $\sim 1.0 \times 10^{21}-1.5 \times 10^{22} \mathrm{~cm}^{-2}$. To explore the dust-to-gas ratios of these bursts, we compare $A_{V}$ with $N_{\mathrm{H}}$ in Figure 13(d). Also plotted in Figure 13(d) are the extinction-to-gas ratios of the MW, LMC, and SMC. ${ }^{12}$ We see that the majority of these bursts have a dust-to-gas ratio smaller than that of the MW, consistent with previous studies (e.g., see Galama \& Wijers 2001; Stratta et al. 2004; Kann et al. 2006). ${ }^{13}$ But $\sim 40 \%$ of the bursts have a dust-to-gas ratio comparable to or higher than that of the LMC. In general, the dust-to-gas ratio derived here is higher than deduced from previous studies which estimated $A_{V}$ from fitting the GRB afterglow SEDs with a SMC-type extinction

\footnotetext{
12 We adopt $N_{\mathrm{H}} / E(B-V) \approx(4.93 \pm 0.28) \times 10^{21},(2 \pm 0.5) \times 10^{22}$, $(4.4 \pm 0.7) \times 10^{22} \mathrm{~cm}^{-2} \mathrm{mag}^{-1}$ and $R_{V} \approx 3.08,3.16$, and 2.93 for the MW (Diplas \& Savage 1994), LMC (Koornneef 1982), and SMC (Bouchet et al. 1985), respectively.

13 Why these GRB hosts have, in general, a lower dust-to-gas ratio than that of the MW? First, these hosts could be metal-poor compared with the MW so that there are fewer heavy elements to make the dust. Second, the bulk of the dust may be really "gray" $(a>1 \mu \mathrm{m})$ so that its presence cannot be revealed by the near-IR-visible-UV extinction curve derived from modeling the afterglow SEDs. The latter explanation is not unreasonable: in the dense dusty circumburst environment, the dust can grow through coagulation to large sizes. Therefore, a lower $A_{V} / N_{\mathrm{H}}$ ratio does not necessarily mean a lower dust-to-gas ratio.
}

Table 3

Dust Size Distributions for the Extinction Curves Derived from the "Drude" Approach and Modeled as a Mixture of Silicate and Graphite Grains

\begin{tabular}{|c|c|c|c|c|c|c|c|}
\hline GRB & $A_{\text {sil }}$ & $\alpha_{\text {sil }}$ & $a_{\mathrm{c}, \text { sil }}(\mu \mathrm{m})$ & $A_{\text {gra }}$ & $\alpha_{\text {gra }}$ & $a_{\mathrm{c}, \text { gra }}(\mu \mathrm{m})$ & $\chi^{2} / N_{\text {obs }}$ \\
\hline 970508 & $1.56 \mathrm{E} 4$ & 2.07 & 0.09 & 7.64 & 2.89 & 0.01 & 0.97 \\
\hline 980703 & $6.17 \mathrm{E} 3$ & 2.13 & 0.11 & $9.73 E-6$ & 3.67 & 0.03 & 0.02 \\
\hline 990123 & $4.50 \mathrm{E}-4$ & 3.44 & 0.73 & 3.83E8 & 1.34 & 0.02 & 1.23 \\
\hline 990510 & 0.03 & 3.26 & 0.04 & 0.10 & 3.00 & 0.95 & 4.20 \\
\hline 991208 & $2.50 \mathrm{E}-4$ & 3.63 & 0.04 & $1.00 \mathrm{E}-3$ & 3.39 & 0.16 & 1.41 \\
\hline 991216 & 0.03 & 3.12 & 0.27 & 1.93 & 2.86 & 0.02 & 1.95 \\
\hline 000911 & 509.19 & 2.33 & 0.12 & $1.20 \mathrm{E}-3$ & 3.40 & 0.02 & 0.04 \\
\hline 010222 & $1.60 \mathrm{E}-4$ & 3.52 & 0.72 & $2.87 \mathrm{E} 9$ & 1.10 & 0.03 & 2.11 \\
\hline 010921 & 0.14 & 3.07 & 0.09 & $7.54 \mathrm{E}-4$ & 3.46 & 0.79 & 2.53 \\
\hline 011121 & 0.01 & 3.30 & 0.14 & $2.00 \mathrm{E}-3$ & 3.24 & 0.68 & 0.07 \\
\hline 020405 & $1.39 \mathrm{E}-4$ & 3.55 & 0.41 & $1.97 \mathrm{E} 8$ & 1.34 & 0.03 & 0.56 \\
\hline 020813 & 0.11 & 3.09 & 0.20 & $1.22 \mathrm{E} 3$ & 2.65 & 0.02 & 3.50 \\
\hline 030226 & $8.85 E-5$ & 3.63 & 0.10 & $2.05 \mathrm{E} 5$ & 1.85 & 0.06 & 2.54 \\
\hline 030328 & 0.03 & 3.19 & 0.07 & 0.08 & 3.00 & 0.83 & 2.89 \\
\hline 030329 & $5.60 \mathrm{E}-5$ & 3.62 & 0.17 & $1.99 \mathrm{E} 3$ & 2.24 & 0.06 & 1.25 \\
\hline 040924 & 0.05 & 3.06 & 0.22 & $3.50 \mathrm{E} 8$ & 1.29 & 0.03 & 0.45 \\
\hline 041006 & $2.40 \mathrm{E}-4$ & 3.43 & 0.03 & 167.7 & 2.47 & 0.06 & 4.13 \\
\hline 050318 & $3.55 \mathrm{E}-3$ & 3.62 & 0.89 & 108.9 & 2.50 & 0.02 & 3.05 \\
\hline 050408 & $4.04 \mathrm{E}-7$ & 3.96 & 0.08 & $5.42 \mathrm{E}-6$ & 3.42 & 0.84 & 4.60 \\
\hline 050525A & 0.01 & 3.20 & 0.39 & 176.0 & 3.16 & 0.02 & 0.23 \\
\hline 050824X & 0.01 & 3.24 & 0.27 & 0.17 & 3.03 & 0.02 & 0.08 \\
\hline 051111 & $9.42 \mathrm{E}-4$ & 3.44 & 0.35 & $5.01 \mathrm{E} 8$ & 1.45 & 0.01 & 0.23 \\
\hline 060614 & 33.61 & 2.55 & 0.14 & $3.56 \mathrm{E} 7$ & 1.42 & 0.03 & 0.06 \\
\hline 060729 & $5.38 \mathrm{E}-9$ & 4.16 & 0.72 & 1.46 & 2.81 & 0.18 & 3.88 \\
\hline 061121 & 0.06 & 3.05 & 0.33 & 0.01 & 3.34 & 0.02 & 0.09 \\
\hline 061126 & 0.10 & 3.02 & 0.19 & 5.27 & 2.65 & 0.08 & 2.22 \\
\hline 070125 & 0.07 & 3.06 & 0.29 & 145.7 & 2.35 & 0.03 & 0.05 \\
\hline 070306 & $9.42 \mathrm{E}-3$ & 3.17 & 1.00 & 0.045 & 2.94 & 0.10 & 4.73 \\
\hline 071003 & $7.08 \mathrm{E}-4$ & 3.40 & 0.64 & 181.7 & 2.44 & 0.04 & 0.75 \\
\hline 080319B & $9.86 \mathrm{E}-5$ & 3.76 & 0.02 & $5.79 \mathrm{E}-4$ & 3.43 & 0.22 & 2.36 \\
\hline 080330 & 0.02 & 3.14 & 0.21 & $1.35 \mathrm{E} 11$ & 0.80 & 0.03 & 1.00 \\
\hline 080514B & $3.69 \mathrm{E}-3$ & 3.27 & 0.47 & 0.81 & 2.80 & 0.08 & 0.80 \\
\hline 081008 & 0.01 & 3.22 & 0.03 & $2.40 \mathrm{E}-3$ & 3.13 & 0.91 & 0.90 \\
\hline
\end{tabular}

law. Finally, the dust-to-gas ratio does not seem to correlate with the shape of the extinction curve inferred from the Drude approach.

We fit the inferred extinction curves using a mixture of spherical amorphous silicate and graphite dust each with an exponential-cutoff power-law size distribution (e.g., see Kim et al. 1994)

$$
\begin{aligned}
A_{\lambda} / A_{V}= & A_{\text {sil }} \int_{a_{\min }}^{a_{\max }} C_{\mathrm{ext}}^{\mathrm{sil}}(a, \lambda) a^{-\alpha_{\text {sil }}} \exp \left(-a / a_{c, \text { sil }}\right) d a \\
& +A_{\text {gra }} \int_{a_{\text {min }}}^{a_{\max }} C_{\text {ext }}^{\text {gra }}(a, \lambda) a^{-\alpha_{\text {gra }}} \exp \left(-a / a_{c, \text { gra }}\right) d a,
\end{aligned}
$$

where the lower (upper) cutoff size $a_{\min }\left(a_{\max }\right)$ is taken to be $50 \AA(1 \mu \mathrm{m})$ for both silicate and graphite dust; the power-law indices $\alpha_{\text {sil }}$ and $\alpha_{\text {gra }}$, and the exponential-cutoff sizes $a_{\mathrm{c}, \text { sil }}$ and $a_{\mathrm{c}, \text { gra }}$ are treated as free parameters; $A_{\text {sil }}$ and $A_{\text {gra }}$ are related to the abundance of each species; and $C_{\mathrm{ext}}^{\text {sil }}\left(C_{\mathrm{ext}}^{\mathrm{gra}}\right)$ is the extinction cross section of silicate (graphite) dust. As shown in Figures 111 , this simple dust model closely fits the extinction curves of all 33 bursts. In Table 3, we present the model parameters.

In Figure 14, we plot the mass fractions of graphite dust of all 33 bursts as a function of redshift. It appears that the relative abundances of graphite to silicate are in general lower than that 
of the MW diffuse ISM ${ }^{14}$ and do not seem to correlate with the redshifts of GRB hosts. In Figure 15, we plot the mean dust sizes of all 33 bursts as a function of redshift. ${ }^{15}$ Again, there is no clear evidence for the dependence of $\langle a\rangle$ on $z$. These issues will be explored in more detail in a subsequent paper (S. L. Liang \& A. Li 2010, in preparation).

\section{CONCLUSION}

We have derived the quantities and wavelength dependencies of the dust extinction along the lines of sight toward 33 nearby GRBs (with $z<2$ ). This is achieved through fitting their afterglow SEDs using the Drude approach. This method does not require a priori assumption of a specific extinction law and therefore allows us to possibly reveal the "true" extinction. The deduced extinction curves display a wide diversity of shapes, ranging from relatively flat curves to curves which are featureless and steeply rise toward the far-UV, and from curves just like that of the MW, LMC, and SMC (which are widely used as extinction templates) to curves resembling that of the MW and the LMC but lacking the $2175 \AA$ bump. The visual extinction $A_{V}$ derived from the Drude approach is generally larger by a factor of $\sim 2-5$ than that inferred by assuming a SMCtype extinction law. The standard silicate-graphite interstellar grain model closely reproduces the extinction curves of all 33 GRBs host galaxies, irrespective of their redshifts. No evidence is found for the evolution of the dust extinction, dust sizes, and relative abundances of silicate to graphite on redshifts.

We thank the anonymous referee for his/her very helpful comments and suggestions. We are supported in part by a NASA/Swift Theory Program, a NASA/Chandra Theory Program, and the NSFC Outstanding Oversea Young Scholarship.

\section{REFERENCES}

Akerlof, C., et al. 1999, Nature, 398, 400

Ballantyne, D. R., et al. 2002, A\&A, 389, L74

Barth, A. J., et al. 2003, ApJ, 584, L47

Berger, E., Cenko, S. B., Fox, D. B., \& Cucchiara, A. 2009, ApJ, 704, 877

Berger, E., \& Mulchaey, J. 2005, GCN Circ., 3122, 1

Beuermann, K., et al. 1999, A\&A, 352, L26

Bloom, J. S. 2005, GCN Circ., 4256, 1

Bloom, J. S., Perley, D. A., \& Chen, H. W. 2006, GCN Circ., 5826, 1

Bloom, J. S., van Dokkum, P. G., Bailyn, C. D., Buxton, M. M., Kulkarni, S.

R., \& Schmidt, B. P. 2004, AJ, 127, 252

Bloom, J. S., et al. 1998, ApJ, 508, L21

Bloom, J. S., et al. 2001, GCN Circ., 1135, 1

Bloom, J. S., et al. 2009, ApJ, 691, 723

Blustin, A. J., et al. 2006, ApJ, 637, 901

Bouchet, P., Lequeux, J., Maurice, E., Prevot, L., \& Prevot-Burnichon, M. L. 1985, A\&A, 149, 330

Butler, N. R., Ricker, G. R., Ford, P. G., Vanderspek, R. K., Marshall, H. L., Jernigan, J. G., Garmire, G. P., \& Lamb, D. Q. 2005, ApJ, 629, 908

Butler, N. R., et al. 2003, ApJ, 597, 1010

Butler, N. R., et al. 2006, ApJ, 652, 1390

14 The silicate-graphite model of Mathis et al. (1977), who assumed a simple power law for the dust size distribution $\left(d n / d a \sim a^{-3.5}\right.$ with

$50 \AA<a<0.25 \mu \mathrm{m}$ for both silicate dust and graphite dust), gives $m_{\text {gra }} /\left(m_{\text {gra }}+m_{\text {sil }}\right) \approx 0.36$. The latest silicate-graphite-polycyclic aromatic hydrocarbon model gives $m_{\text {gra }} /\left(m_{\text {gra }}+m_{\text {sil }}\right) \approx 0.27$ (Weingartner \& Draine 2001). The latter model assumes two different size distributions which smoothly extend from a few angstroms to a few micrometers for silicate dust and graphitic dust.

15 The mean dust sizes are derived by first averaging over the silicate (graphite) size distributions weighted by mass to obtain $\langle a\rangle_{\text {sil }}\left(\langle a\rangle_{\text {gra }}\right)$ and then taking the mass-weighted average of $\langle a\rangle_{\text {sil }}$ and $\langle a\rangle_{\text {gra }}$ :

$\langle a\rangle=m_{\text {sil }} /\left(m_{\text {gra }}+m_{\text {sil }}\right) \times\langle a\rangle_{\text {sil }}+m_{\text {gra }} /\left(m_{\text {gra }}+m_{\text {sil }}\right) \times\langle a\rangle_{\text {gra }}$.
Calzetti, D., Kinney, A. L., \& Storchi-Bergmann, T. 1994, ApJ, 429, 582

Cardelli, J. A., Clayton, G. C., \& Mathis, J. S. 1989, ApJ, 345, 245

Castro-Tirado, A. J., et al. 1999, ApJ, 511, L85

Castro-Tirado, A. J., et al. 2001, A\&A, 370, 398

Cenko, S. B., et al. 2009, ApJ, 693, 1484

Chary, R., Becklin, E. E., \& Armus, L. 2002, ApJ, 566, 229

Chary, R., et al. 1998, ApJ, 498, L9

Chen, S. L., Li, A., \& Wei, D. M. 2006, ApJ, 647, L13

Cobb, B. E. 2008a, GCN Circ., 7553, 1

Cobb, B. E. 2008b, GCN Circ., 8356, 1

Cobb, B. E., \& Bailyn, C. D. 2005, GCN Circ., 3506, 1

Covino, S., et al. 2003, A\&A, 404, L5

Dado, S., Dar, A., De Rújula, A., \& Plaga, R. 2008, ApJ, 678, 353

D’Avanzo, P., D'Elia, V., \& Covino, S. 2008, GCN Circ., 8350, 1

Della Valle, M., et al. 2006a, ApJ, 642, L103

Della Valle, M., et al. 2006b, Nature, 444, 1050

de Ugarte Postigo, A., et al. 2007, A\&A, 462, L57

Diplas, A., \& Savage, B. D. 1994, ApJ, 427, 274

Djorgovski, S. G., Dierks, A., Bloom, J. S., Kulkarni, S. R., Filippenko, A. V., Hillenbrand, L. A., \& Carpenter, J. 1999, GCN Circ., 481, 1

Djorgovski, S. G., Frail, D. A., Kulkarni, S. R., Bloom, J. S., Odewahn, S. C., \& Diercks, A. 2001, ApJ, 562, 654

Djorgovski, S. G., et al. 1998, ApJ, 508, L17

Dodonov, S. N., Afanasiev, V. L., Sokolov, V. V., Moiseev, A. V., \& CastroTirado, A. J. 1999, GCN Circ., 475, 1

Draine, B. T. 2003, ARA\&A, 41, 241

Draine, B. T., \& Hao, L. 2002, ApJ, 569, 780

Elíasdóttir, Á., et al. 2009, ApJ, 697, 1725

Fitzpatrick, E. L., \& Massa, D. 1990, ApJS, 72, 163

Flasher, J., et al. 2005a, GCN Circ., 3561, 1

Flasher, J., et al. 2005b, GCN Circ., 3567, 1

Foley, R. J., Chen, H.-W., Bloom, J., \& Prochaska, J. X. 2005, GCN Circ., 3483,1

Foley, R. J., et al. 2006, ApJ, 645, 450

Fox, D. B., Berger, E., Price, P. A., \& Cenko, S. B. 2007, GCN Circ., 6071, 1

Fynbo, J. P. U., et al. 2005, GCN Circ., 3874, 1

Fynbo, J. P. U., et al. 2007, Messenger, 130, 43

Galama, T. J., \& Wijers, R. A. M. J. 2001, ApJ, 549, L209

Galama, T. J., et al. 1998, ApJ, 497, L13

Galama, T. J., et al. 1999, Nature, 398, 394

Galama, T. J., et al. 2003, ApJ, 587, 135

Garnavich, P. M., Jha, S., Pahre, M. A., Stanek, K. Z., Kirshner, R. P., Garcia, M. R., Szentgyorgyi, A. H., \& Tonry, J. L. 2000, ApJ, 543, 61

Garnavich, P. M., et al. 2003, ApJ, 582, 924

Gomboc, A., et al. 2008, ApJ, 687, 443

Gordon, K. D., \& Clayton, G. C. 1998, ApJ, 500, 816

Gordon, K. D., Clayton, G. C., Misselt, K. A., Landolt, A. U., \& Wolff, M. J. 2003, ApJ, 594, 279

Gorosabel, J., et al. 2005, A\&A, 444, 711

Greiner, J., Guenther, E., Klose, S., \& Schwarz, R. 2003a, GCN Circ., 1886, 1

Greiner, J., Peimbert, M., Estaban, C., Kaufer, A., Jaunsen, A., Smoke, J., Klose, S., \& Reimer, O. 2003b, GCN Circ., 2020, 1

Greiner, J., et al. 2003c, ApJ, 599, 1223

Greiner, J., et al. 2008, PASP, 120, 405

Groot, P. J., et al. 1998, ApJ, 493, L27

Grupe, D., et al. 2006, GCN Circ., 5365, 1

Grupe, D., et al. 2007, ApJ, 662, 443

Halpern, J. P., et al. 2000, ApJ, 543, 697

Hill, G., Prochaska, J. X., Fox, D., Schaefer, B., \& Reed, M. 2005, GCN Circ., 4255,1

Hjorth, J., et al. 2003, ApJ, 597, 699

Infante, L., Garnavich, P. M., Stanek, K. Z., \& Wyrzykowski, L. 2001, GCN Circ., 1152, 1

Jakobsson, P., Hjorth, J., Fynbo, J. P. U., Watson, D., Pedersen, K., Björnsson, G., \& Gorosabel, J. 2004, ApJ, 617, L21

Jaunsen, A. O., et al. 2008, ApJ, 681, 453

Jha, S., et al. 2001, ApJ, 554, L155

Kahharov, B., Ibrahimov, M., Sharapov, D., Pozanenko, A., Rumyantsev, V., \& Beskin, G. 2005, GCN Circ., 3261, 1

Kann, D. A., Klose, S., \& Zeh, A. 2006, ApJ, 641, 993

Kann, D. A., et al. 2007, arXiv:0712.2186

Kaplan, D., Garnavich, P., Rosenberg, J., \& Stanek, K. Z. 2005, GCN Circ., 3507, 1

Kim, S.-H., Martin, P. G., \& Hendry, P. D. 1994, ApJ, 422, 164

Kinney, A. L., Calzetti, D., Bohlin, R. C., McQuade, K., Storchi-Bergmann, T., \& Schmitt, H. R. 1996, ApJ, 467, 38 
Klose, S., et al. 2000, ApJ, 545, 271

Klose, S., et al. 2004, AJ, 128, 1942

Klotz, A., Boër, M., Atteia, J. L., Stratta, G., Behrend, R., Malacrino, F., \& Damerdji, Y. 2005, A\&A, 439, L35

Koornneef, J. 1982, A\&A, 107, 247

Krühler, T., et al. 2008, ApJ, 685, 376

Larson, K. A., Whittet, D. C. B., \& Hough, J. H. 1996, ApJ, 472 755

Lazzati, D., et al. 2001, A\&A, 378, 996

Lee, B. C., et al. 2001, ApJ, 561, 183

Levan, A. J., et al. 2006, ApJ, 648, L9

Li, A., \& Greenberg, J. M. 1998, A\&A, 339, 591

Li, A., Liang, S. L., Kann, D. A., Wei, D. M., Klose, S., \& Wang, Y. J. 2008a, ApJ, 685,1046

Li, Y., Li, A., \& Wei, D. M. 2008b, ApJ, 678, 1136

Liang, S. L., \& Li, A. 2009, ApJ, 690, L56

Maiolino, R., Schneider, R., Oliva, E., Bianchi, S., Ferrara, A., Mannucci, F., Pedani, M., \& Roca Sogorb, M. 2004, Nature, 431, 533

Maiorano, E., et al. 2006, A\&A, 455, 423

Malesani, D., Fynbo, J. P. U., Jakobsson, P., Vreeswijk, P. M., \& Niemi, S.-M. 2008, GCN Circ., 7544, 1

Mangano, V., et al. 2007, A\&A, 470, 105

Martini, P., Garnavich, P., \& Stanek, K. Z. 2003, GCN Circ., 1980, 1

Masetti, N., Palazzi, E., Pian, E., Hjorth, J., Castro-Tirado, A., Boehnhardt, H., \& Price, P. 2002, GCN Circ., 1330, 1

Masetti, N., et al. 2001, A\&A, 374, 382

Masetti, N., et al. 2003, A\&A, 404, 465

Masetti, N., et al. 2005, A\&A, 438, 841

Mathis, J. S., Rumpl, W., \& Nordsieck, K. H. 1977, ApJ, 217, 425

Melandri, A., et al. 2006, GCN Circ., 5827, 1

Melandri, A., et al. 2008, ApJ, 686, 1209

Metzger, M. R., et al. 1997a, IAU Circ., 6676, 3

Metzger, M. R., et al. 1997b, Nature, 387, 879

Michałowski, M. J., Hjorth, J., Castro Cerón, J. M., \& Watson, D. 2008, ApJ, 672,817

Michałowski, M. J., et al. 2009, ApJ, 693, 347

Milne, P. A., Williams, G. G., \& Park, H.-S. 2005, GCN Circ., 3258, 1

Mirabal, N., et al. 2003, ApJ, 587, 128

Misselt, K. A., Clayton, G. C., \& Gordon, K. D. 1999, ApJ, 515, 128

Page, K. L., et al. 2007, ApJ, 663, 1125

Perley, D. A., et al. 2008a, ApJ, 672, 449

Perley, D. A., et al. 2008b, ApJ, 688, 470

Perley, D. A., et al. 2009, AJ, 138, 1690

Perna, R., Lazzati, D., \& Fiore, F. 2003, ApJ, 585, 775

Perri, M., et al. 2005, A\&A, 442, L1

Press, W. H., Teukolsky, S. A., Vetterling, W. T., \& Flannery, B. P. 1992, Numerical Recipes in FORTRAN: The Art of Scientific Computing (2nd ed.; Cambridge: Cambridge Univ. Press)

Price, P. A., Berger, E., \& Fox, D. B. 2006, GCN Circ., 5275, 1

Price, P. A., Bloom, J. S., Goodrich, R. W., Barth, A. J., Cohen, M. H., \& Fox, D. W. 2002c, GCN Circ., 1475, 1

Price, P. A., Roth, K., Rich, J., Schmidt, B. P., Peterson, B. A., Cowie, L., Smith, C., \& Rest, A. 2004, GCN Circ., 2791, 1
Price, P. A., et al. 2002a, ApJ, 573, 85

Price, P. A., et al. 2002b, ApJ, 571, L121

Price, P. A., et al. 2003, ApJ, 584, 931

Prochaska, J. X., Bloom, J. S., Chen, H.-W., Foley, R. J., \& Roth, K. 2005, GCN Circ., 3204, 1

Prochaska, J. X., et al. 2009, ApJ, 691, L27

Racusin, J. L., Schady, P., \& Palmer, D. 2008, GCN Rep., 173, 1

Reichart, D. E. 1998, ApJ, 495, L99

Reichart, D. E. 2001, ApJ, 553, 235

Rossi, A., et al. 2008, A\&A, 491, L29

Savage, B. D., Massa, D., Meade, M., \& Wesselius, P. R. 1985, ApJS, 59, 397

Savaglio, S., \& Fall, S. M. 2004, ApJ, 614, 293

Savaglio, S., Fall, S. M., \& Fiore, F. 2003, ApJ, 585, 638

Schady, P., et al. 2007, MNRAS, 377, 273

Silvey, J., et al. 2004, GCN Circ., 2833, 1

Soderberg, A. M., et al. 2006, ApJ, 636, 391

Sollerman, J., et al. 2007, A\&A, 466, 839

Stanek, K. Z., Garnavich, P. M., Kaluzny, J., Pych, W., \& Thompson, I. 1999, ApJ, 522, L39

Starling, R. L. C. 2008, A\&A, 488, 915

Starling, R. L. C., Wijers, R. A. M. J., Wiersema, K., Rol, E., Curran, P. A., Kouveliotou, C., van der Horst, A. J., \& Heemskerk, M. H. M. 2007, ApJ, 661,787

Still, M., et al. 2005, ApJ, 635, 1187

Stratta, G., Fiore, F., Antonelli, L. A., Piro, L., \& De Pasquale, M. 2004, ApJ, 608,846

Stratta, G., Maiolino, R., Fiore, F., \& D’Elia, V. 2007, ApJ, 661, L9

Stratta, G., Perna, R., Lazzati, D., Fiore, F., Antonelli, L. A., \& Conciatore, M L. 2005, A\&A, 441, 83

Tanvir, N. R., et al. 2008, MNRAS, 388, 1743

Thoene, C. C., et al. 2006, GCN Circ., 5373, 1

Updike, A. C., et al. 2008, ApJ, 685, 361

van der Horst, A. J., Kouveliotou, C., Gehrels, N., Rol, E., Wijers, R. A. M. J., Cannizzo, J. K., Racusin, J., \& Burrows, D. N. 2009, ApJ, 699, 1087

Vreeswijk, P. M., Smette, A., Malesani, D., Fynbo, J. P. U., Milvang-Jensen, B., Jakobsson, P., Jaunsen, A. O., \& Ledoux, C. 2008, GCN Circ., 7444, 1

Vreeswijk, P. M., et al. 1999a, ApJ, 523, 171

Vreeswijk, P. M., et al. 1999b, GCN Circ., 324, 1

Vreeswijk, P. M., et al. 1999c, GCN Circ., 496, 1

Vreeswijk, P. M., et al. 2006, A\&A, 447, 145

Waxman, E., \& Draine, B. T. 2000, ApJ, 537, 796

Weingartner, J. C., \& Draine, B. T. 2001, ApJ, 548, 296

Wiersema, K., Rol, E., Starling, R., Tanvir, N., Bloomfield, D. S., \& Thompson, H. 2005, GCN Circ., 3699, 1

Wiersema, K., Starling, R. L. C., Rol, E., Vreeswijk, P., \& Wijers, R. A. M. J. 2004, GCN Circ., 2800, 1

Wijers, R. A. M. J., \& Galama, T. J. 1999, ApJ, 523, 177

Woosley, S. E., \& Bloom, J. S. 2006, ARA\&A, 44, 507

Yanagisawa, K., Toda, H., \& Kawai, N. 2005, GCN Circ., 3489, 1

Yost, S. A., Schaefer, B. E., \& Yuan, F. 2006, GCN Circ., 5824, 1

Yost, S. A., et al. 2007, ApJ, 657, 925

Zheng, W.-K., Deng, J.-S., \& Wang, J. 2009, Res. Astron. Astrophys., 9, 1103

Zubko, V., Dwek, E., \& Arendt, R. G. 2004, ApJS, 152, 211 